ACTA ASTRONOMICA

Vol. 59 (2009) pp. 137-167

\title{
Galactic Fundamental Mode RR Lyrae Stars. Period-Amplitude Diagram, Metallicities and Distribution
}

\author{
by \\ D. M. Szczygieł, G. Pojmański and B. Pilecki \\ Warsaw University Observatory, Al. Ujazdowskie 4, 00-478 Warsaw, Poland \\ e-mail: (dszczyg, gp, pilecki)@astrouw.edu.pl
}

Received June 8, 2009

\begin{abstract}
We have analyzed 1455 fundamental mode RR Lyr stars of the Galactic field, using the All Sky Automated Survey (ASAS) data. The sample covers $75 \%$ of the sky and contains objects in the close neighborhood of the Sun, within $4 \mathrm{kpc}$ distance. Unlike in the previous analysis of the close field RRab stars, we see a clear manifestation of the Oosterhoff groups on the period-amplitude diagram. The relation for Oosterhoff I type variables becomes strongly flattened at large $V$ amplitudes, which was not observed for globular cluster RR Lyr. We calculate photometric metallicities using two available methods: one of Jurcsik and Kovács (1996) and the other of Sandage (2004). We find significant discrepancies between results from both methods. Comparison with spectroscopic metallicities undoubtedly favors the method of Jurcsik and Kovács (1996). In addition, we notice that RRab stars of Oosterhoff II type might follow a different metallicity-period-phase relation than Oosterhoff I type variables. The spatial distribution of Galactic field RRab stars does not show any metallicity gradients with distance from the Galactic center in either of the Oosterhoff groups. However, both the older, metal poor Oosterhoff II variables and the metal rich Oosterhoff I RRab stars become more concentrated to the Galactic plane with increasing metal content.
\end{abstract}

Key words: Stars: Population II - Stars: variables: RR Lyr-Stars: abundances - Stars: fundamental parameters

\section{Introduction}

RR Lyr type stars are radially pulsating giants of the horizontal branch on the HR diagram. Their periods are in the range of 0.2-1.2 days and the amplitudes of light variations are typically $0.2-1.6 \mathrm{mag}$ in the $V$-band. Their almost constant absolute mean magnitude (about 0.6 in $V$ ) makes them good distance indicators and their advanced age (over $10 \mathrm{Gyrs}$ ) means they represent a chemical composition of the early stages of Galaxy formation. Therefore RR Lyr stars are excellent objects for investigating the structure and composition of the early nearby Universe. 
Since the first detection of RR Lyr stars in globular clusters, their number has increased rapidly over the last decade thanks to many sky surveys. There are approximately 3000 RR Lyr stars known in the field of the Milky Way within the radius of $\approx 4 \mathrm{kpc}$ from the Sun, identified in the ASAS and NSVS catalogs (Pojmański 2002, Kinemuchi et al. 2006, Wils, Lloyd and Bernhard 2006), almost 4000 in globular clusters and the halo (Miceli et al. 2008), 2800 in the Galactic bulge (Mizerski 2003). Recently, an impressing number of 25000 RR Lyr stars was identified in the OGLE data of Large Magellanic Cloud by Soszyński et al. (2009).

An interesting phenomenon concerning RR Lyr variables is the Oosterhoff dichotomy (Oosterhoff 1939) observed in globular clusters of the Milky Way and absent in other nearby galaxies. The dichotomy is believed to originate from the metallicity and age differences (Lee and Carney 1999, Clement and Shelton 1999, Cacciari, Corwin and Carney 2005), but the final explanation still has not been constructed.

In their paper on Galactic field RR Lyr stars Kinemuchi et al. (2006) addressed a problem of Oosterhoff dichotomy for field RRab stars, which has been a matter of argument since the first work of Suntzeff, Kinman and Kraft (1991) who showed the existence of dichotomy among the Galactic field RRab stars. Kinemuchi et al. (2006) found that there is no clear separation in the period-amplitude diagram as observed in the Galactic halo. That is, the Oosterhoff dichotomy is not a property of a whole Galaxy, but only of its outer parts. Such conclusion puts important constraints on the early evolution of the Galaxy and in this paper we attempt to verify this result by utilizing the Galactic field data from the All Sky Automated Survey.

In Section 2 we describe the data, in Section 3 we investigate the periodamplitude diagram and color properties of ASAS RRab stars in order to verify the existence of Oosterhoff dichotomy. Section 4 presents the metallicity analysis of ASAS RRab stars and a detailed comparison of two methods of photometric metallicity determination. In Section 5 we investigate the Galactic distribution and we summarize the results in Section 6. The Appendix presents the detailed comparison of ASAS and NSVS catalogs thus explaining discrepancies between some of the results.

\section{RR Lyr Data}

\subsection{The ASAS Project}

The All Sky Automated Survey 1 (ASAS) is an ongoing project dedicated to whole sky monitoring and variability detection. Its southern observing station consists of two small wide-field telescopes (200/2.8) located at Las Campanas Obser-

\footnotetext{
${ }^{1}$ http://www.astrouw.edu.pl/asas
} 
vatory in Chile, equipped with standard $V$ and $I$ filters, which have been observing the whole available sky south of $+28^{\circ}$ since 2000 (and some parts since 1997). ASAS has identified and classified over 50000 variable stars brighter than $14.5 \mathrm{mag}$ in the $V$-band and published the results in the form of the catalog (Pojmański 2002, 2003, Pojmański and Maciejewski 2004, 2005, Pojmański, Pilecki and Szczygieł 2005). The ASAS Catalog of Variable Stars (hereafter ACVS) contains information on star's coordinates, $V$ magnitude, $V$ amplitude, period of variation, 2MASS colors and variability type. All light curves are available for download and typically contain 300-500 good quality points. The number of RR Lyr stars pulsating in the fundamental mode available in ACVS is 1455. Their distribution in Galactic coordinates is presented in Fig. 1.

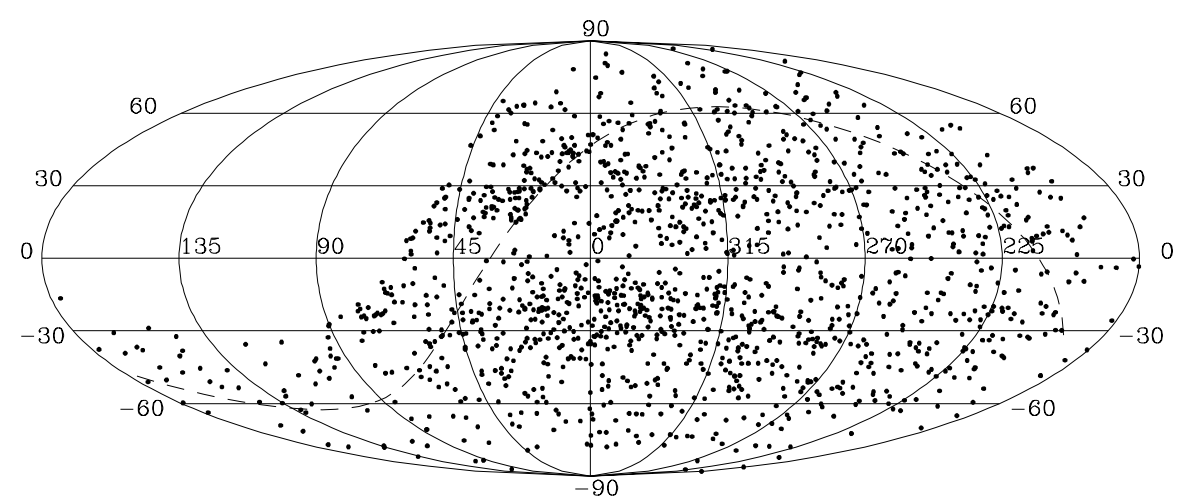

Fig. 1. Distribution of 1455 fundamental mode RR Lyr stars from the ASAS Catalog of Variable Stars (ACVS) in Galactic coordinates. The dashed line is the equatorial plane.

\subsection{V and I-Band Light Curve Preparation}

The $V$-band light curves of all 1455 RRab stars in ACVS were extracted from the catalog and only points with quality flags 'A' and 'B' were retained. All light curves were subject to preliminary cleaning and frequency analysis in order to refine their pulsation period values, which is especially useful in the case of objects from older parts of the catalog, when less data points were available (R.A. 0-6h). The light curves of stars for which new and old periods differed by more than $10 \%$ were visually examined and the proper period was assigned. A few of them turned out to be other type variables, usually Cepheids or contact binaries, a few had poor quality light curves and their variability type was hard to define. At this stage a chosen period was fixed for the following analysis. The number of stars was reduced to 1437.

Then an iterative cleaning process was performed. First, all points deviating more than $3 \sigma$ were removed. Then a 6 harmonic sine Fourier model was fitted 
to the light curve and again all points deviating by more than $3 \sigma$ were removed. This step was repeated once more. Afterward, a 6 harmonic model was subtracted from the light curve and a linear trend was subtracted. Then the Fourier model was restored and once again we performed a 6 harmonic fit but now without a linear trend. Finally, we cleaned the light curve for the last time and restored the linear model.

The ASAS light curve catalogs contain only $V$-band observations, but ASAS was observing simultaneously in the $I$-band also. The $I$-band data has recently been reduced and will soon be publicly available. For the purpose of this paper we extracted light curves of RR Lyr stars in order to obtain I magnitudes and amplitudes. The average number of good observations (flags 'A' and 'B') per star is around 150-250, but there were some objects with less than 30 measurements and those were rejected from further analysis. All 1423 remaining light curves were cleaned in the same way as in the $V$-band.

After the preparation process we once again fitted a sine Fourier series to all phased $V$ - and $I$-band light curves independently, in the form:

$$
m=a_{0}+\sum_{i=1}^{N} a_{i} \sin \left(2 \pi i x+\varphi_{i}\right)
$$

where $x=\left(\mathrm{HJD}-\mathrm{HJD}_{0}\right) / P$ and $P$ is the pulsation period. $\mathrm{HJD}_{0}$ stands for the epoch of maximum brightness. The number of harmonics $N$ was set individually for each star, using a slightly modified method of Kovács (2005) and it varied from 6 to 10, depending on the signal to noise ratio (smaller order of the fit for more noisy light curves). For each of 1423 RRab variables all Fourier parameters $a_{0} \ldots a_{N}$ and $\varphi_{1} \ldots \varphi_{N}$ extracted from more numerous $V$-band light curves were stored as well as mean and maximum brightness magnitudes and amplitudes of variation, in both bands. They are available for download from the ASAS website. A few exemplary lines of the file containing Fourier parameters are presented in Table 1.

\section{Period, Amplitude and Color of ASAS RRab Variables}

Fig. 2 shows three histograms of RRab variables: the distribution of $V$-band magnitudes, amplitudes and periods. ASAS limiting magnitude is roughly $V=$ $14 \mathrm{mag}$, putting a distance limit at $4.5 \mathrm{kpc}$ but a limiting magnitude at which detection efficiency drops significantly is $13.8 \mathrm{mag}$, thus we reject all objects fainter than that. This leaves us with 1227 stars. The $V$-band amplitudes of ASAS RRab stars are in the range of $0.16-1.5 \mathrm{mag}$ and are defined as a difference between the maximum and the minimum value of the light curve model as described by Eq. (1). There is a visibly lower number of variables with amplitudes smaller than $0.5 \mathrm{mag}$, which is an effect of lower detection efficiency. The mean amplitude value is 0.88 mag. Pulsation periods are in the range of 0.2 to 1.2 days with the mean 
Table 1

Fourier parameters of $V$-band light curves

\begin{tabular}{ccccccccc}
\hline ID & $\mathrm{N}$ & $a_{0}$ & $a_{0, \mathrm{err}}$ & $a_{1}$ & $a_{1, \mathrm{err}}$ & $\varphi_{1}$ & $\varphi_{1, \mathrm{err}}$ & $\ldots$ \\
\hline $000036+2639.8$ & 6 & 13.218 & 0.019 & 0.331 & 0.026 & 4.078 & 0.086 & $\ldots$ \\
$000248-2456.7$ & 10 & 10.297 & 0.002 & -0.403 & 0.002 & 0.825 & 0.005 & $\ldots$ \\
$000301-7041.5$ & 6 & 13.707 & 0.011 & 0.333 & 0.015 & 4.228 & 0.048 & $\ldots$ \\
\hline
\end{tabular}

First three lines of the file containing Fourier parameters for 1423 ASAS RRab stars. The columns contain the ASAS ID of a star, number of harmonics $N$, and a series of Fourier parameters with their errors, from 0 to $N$ : $a_{0}, a_{0, \mathrm{err}}, a_{1}, a_{1, \mathrm{err}}, \varphi_{1}, \varphi_{1, \mathrm{err}}, \ldots, a_{N}, a_{N, \mathrm{err}}, \varphi_{N}, \varphi_{N, \mathrm{err}}$. The complete file is available for download from the ASAS website.

value of 0.56 days, very close to 0.55 days which is an average period value for Oosterhoff I type globular cluster RRab stars.
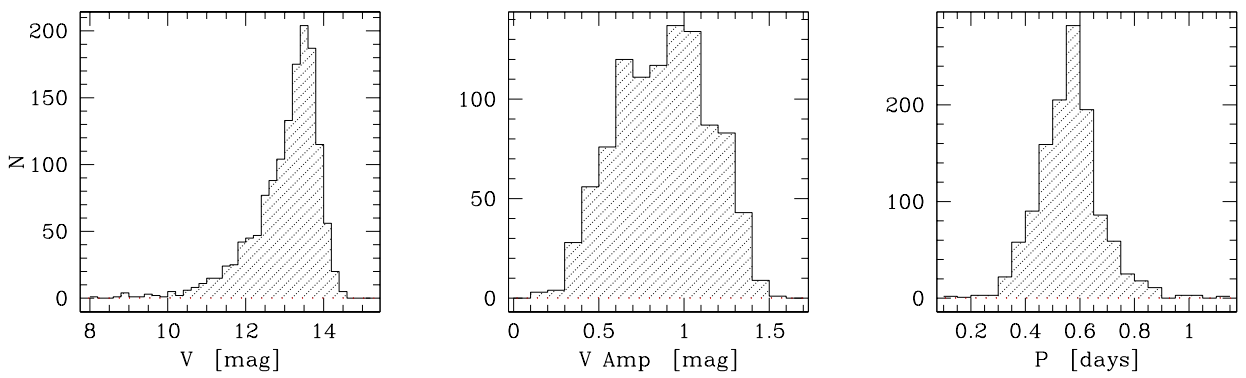

Fig. 2. Distributions of $V$-band average magnitudes (left panel), amplitudes (middle panel) and pulsation periods (right panel) for 1423 RRab stars from ACVS.

\subsection{The Period-Amplitude Diagram}

Soon after the extensive works of Bailey and Pickering (1913) on cluster variables it has been discovered that globular clusters of our Galaxy fall into two distinct groups according to their RR Lyr mean pulsation periods and a number of RRc variables relative to number of RRab stars. These two groups had been called Oosterhoff I and II (Oosterhoff 1939) and we will refer to them as Oo I and Oo II. Mean periods of RRab pulsators are $\left\langle P_{a b}\right\rangle=0.55 \mathrm{~d}$ (for Oo I) and $\left\langle P_{a b}\right\rangle=0.64 \mathrm{~d}$ (for Oo II) and the number of RRc stars relative to the number of RRab stars is smaller for Oo I $\left(N_{c} / N_{a b}=0.17\right)$ than for Oo II $\left(N_{c} / N_{a b}=0.44\right)$. It was also noticed that these two groups possess different metallicities, with Oo I clusters being more metal rich than Oo II. In the later studies it became clear that while the separation into Oosterhoff groups is significant, there is a continuum of period and metallicity 
values within each group (Sandage 1982).

When plotted in the log period-amplitude plane, RRab stars from different Oosterhoff clusters follow two separate parallel relations such that longer period stars have smaller amplitudes, and for a given amplitude value Oo II RRab stars have longer periods. There have been many attempts to explain this phenomenon and the research history is well described in the work of Lee and Carney (1999). For now it is agreed that the main cause of this period shift is the luminosity difference caused by a different evolutionary state of RR Lyr variables - RRab stars that follow period-amplitude relation typical of Oo II globular clusters are believed to be more evolved than the ones following Oo I relation (Lee and Carney 1999, Clement and Shelton 1999, Cacciari, Corwin and Carney 2005).

The situation is somewhat different in the case of Galactic halo RR Lyr stars. As summarized by Catelan (2009), there has been no agreement whether the Oosterhoff dichotomy is present in the field of the Galaxy or not. In the first works regarding this subject, Suntzeff et al. (1991) showed that it does exist, but later Vivas and Zinn (2003) proved the opposite using the QUEST data. Recently Kinemuchi et al. (2006) constructed a period-amplitude (Bailey) diagram for about 600 close field RRab stars, within the distance of $4.5 \mathrm{kpc}$ from the Sun, using data of the Northern Sky Variability Survey 2 (NSVS). In their Fig. 12 the authors show that there are no clear Oosterhoff groups visible on the diagram and there is rather a continuum of periods and amplitudes within their sample, although the majority of RRab stars are located close to the region occupied by Oo I globular cluster RR Lyr stars. The same diagram was plotted for over 800 RRab stars of the Galactic halo (excluding globular cluster variables), spanning the distances from about 4 to $20 \mathrm{kpc}$ from the Sun (Miceli et al. 2008), based on data collected by the Lowell Observatory Near Earth Objects Survey Phase I (LONEOS-I). Here, the majority of RR Lyr variables are undeniably concentrated in the regions corresponding to those occupied by Oosterhoff groups in globular clusters, especially in the region of Oo I group.

The resulting picture is that RRab variables from the Galactic halo display the separation into Oosterhoff groups, typical of globular clusters, while very close field RRab stars do not. According to Lee and Carney (1999) the age differences between Oo I and Oo II groups are about 2-3 Gyrs (Oo I being younger), which implies not only different formation times, but possibly a different origin of Oo I and Oo II RR Lyr stars and sheds some light on the possible formation history scenarios. In particular, Oo II type clusters are believed to have formed early in the proto Galaxy formation stage, and Oo I type 2-3 Gyrs later. Also, some Oo I clusters could have joined the Galaxy by accretion processes (van den Bergh 1993), but this hypothesis has been recently questioned, since RR Lyr stars in neighboring dwarf galaxies do not display the Oosterhoff dichotomy, but quite the contrary they fall into the Oosterhoff gap on the Bailey diagram. Absence of the Oosterhoff

\footnotetext{
${ }^{2}$ http://skydot.lanl.gov/nsvs/nsvs.php
} 
effect in Sun's proximity would imply that there was rather a continuous creation rate of Galactic RRab stars, without particular "events" such as accretion processes, at least in regions close to the Galactic plane. Another scenario could allow for the accretion processes to add up to the Galactic halo content.

We construct a Bailey diagram for the ASAS fundamental mode RR Lyr stars in Fig. 3. There is a continuum of periods and amplitudes, but unlike in the NSVS sample, we see a clear manifestation of the Oosterhoff groups with the majority of objects lying in the Oo I group region. Given the similarity of ASAS and ROTSE-I telescopes, the ACVS and NSVS catalogs have practically the same angular resolution, magnitude range and thus distance limit to RR Lyr stars. In this case we would expect them to have uniform RR Lyr samples exhibiting same properties, which is not observed on the Bailey diagram. In order to check the conformity of both catalogs we investigated RRab variables in the overlapping region of ASAS and NSVS, between declinations $-38^{\circ} \div+28^{\circ}$. We found that corresponding objects from ASAS and NSVS catalogs often have significantly different amplitudes, even up to 0.5 mag. Thus, different distributions in period-amplitude diagram most probably originate from wrongly determined amplitudes of NSVS RR Lyr stars, possibly a result of unfiltered ROTSE observations. The details of ASAS and NSVS catalogs comparison are presented in the Appendix.
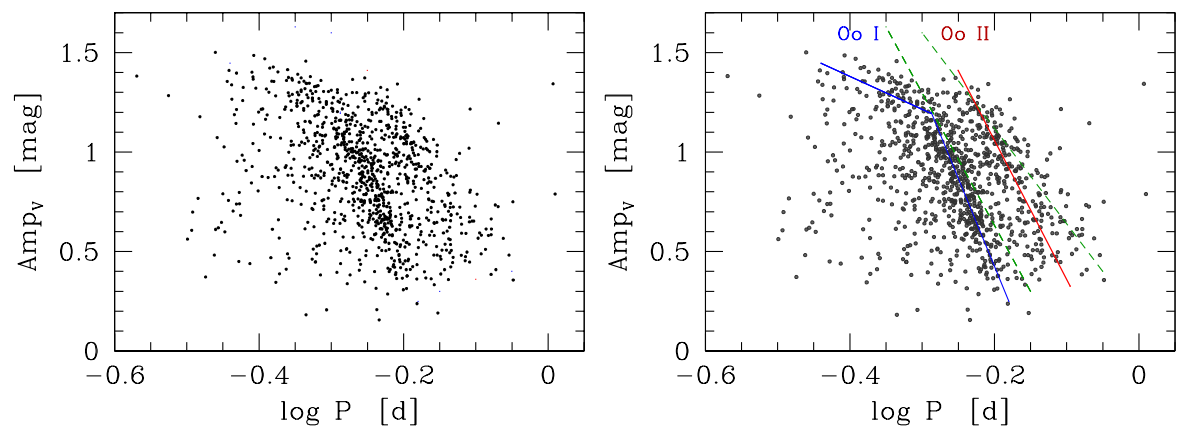

Fig. 3. Both panels present a period-amplitude (Bailey) diagram for ASAS RRab stars. The green dashed lines overplotted on the right panel image represent relations for M3 (Oo I globular cluster) and $\omega$ Cen (Oo II globular clusters) derived by Clement and Rowe (2000). The solid lines are the fits to Oo I (blue) and Oo II (red) groups derived in the course of this work.

For reference, in the right panel of Fig. 3 we overplot the Oosterhoff relations for Oo I and Oo II globular clusters used by Kinemuchi et al. (2006) in the NSVS plots (their Figs. 12, 13 and 26), and derived by Clement and Rowe (2000) for clusters M3 (Oo I) and $\omega$ Cen (Oo II). It is well visible that these relations are somewhat shifted with reference to the main concentration of ASAS data points. That is, at constant amplitude value ASAS Oosterhoff groups have shorter average periods than globular cluster Oosterhoff groups. Since this $\log P$ shift is explained as the difference in age and/or evolutionary advancement this would imply that the evolutionary advancement is different among field RRab stars of the two Oosterhoff 
types and typical globular cluster RRab stars. If the Oosterhoff concentrations can be credited to some episodes in the Galaxy formation (accretion from neighboring galaxies has been suggested) then their different characteristics among ASAS RRab stars means that some other processes took place in the proximity of the Galactic plane. Of course there is a continuum of points on the Bailey diagram indicating that there was an RR Lyr stars production at all times of Galaxy formation. The same plot constructed for a subsample of bright RR Lyr stars with well defined amplitudes does not change the location and the shape of the two Oosterhoff relations.

The period-amplitude relation for Oosterhoff I type variables becomes strongly flattened at large $V$ amplitude values, which was not observed for globular cluster RR Lyr before. While it is true that Cacciari et al. (2005) noticed that a quadratic relation provides a better fit to their data for Oo I type cluster M3, the curvature of their relation is small. In the case of ASAS RRab stars the best fit to Oo I group is a fourth order polynomial, but we choose a much simpler two line fit, as the relation becomes strongly rather than smoothly flattened at high amplitudes:

$$
\begin{array}{ll}
A m p_{\mathrm{V}}=-8.844 \times \log P-1.343 & \mathrm{Amp}_{\mathrm{V}}<1.2, \\
\mathrm{Amp}_{\mathrm{V}}=-1.654 \times \log P+0.719 & \mathrm{Amp} \mathrm{V} \geq 1.2 .
\end{array}
$$

The above relations are overplotted in Fig. 3 with blue solid line. The reason that this "flattening" effect was not observed before might indicate that there is a lack of high amplitude RRab satrs in globular clusters. Another possible reason is small number statistics for a given cluster. We also checked that this effect is not due to wrongly determined RR Lyr stars amplitudes.

The relation for Oosterhoff II type RRab stars remains simple, being a one linear equation of the form (red solid line in Fig. 3):

$$
\mathrm{Amp}_{\mathrm{V}}=-7.007 \times \log P-0.343 .
$$

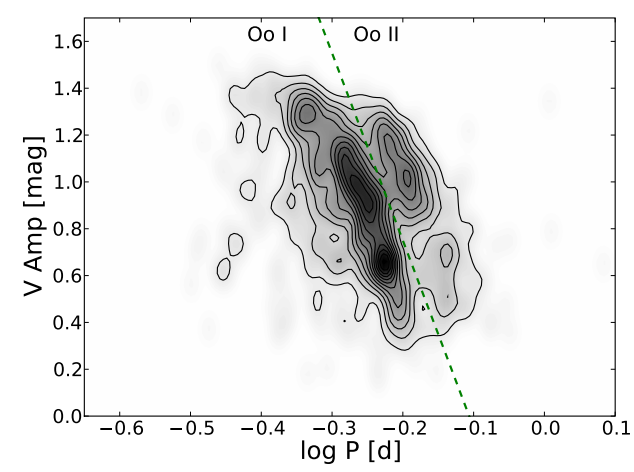

Fig. 4. Density maps of the period-amplitude (Bailey) diagram for ASAS RRab stars. The dashed line separates Oo I and Oo II type variables and it follows Eq. (5).

We draw density maps of the Bailey diagram in Fig. 4 to show that the highest concentration of RRab stars in Oosterhoff I group indeed follows a relation of different shape than in the case of globular cluster or distant halo RRab stars. As for 
Oosterhoff II group it seems that the relation is linear although there are no high amplitude Oo II type RRab stars. Another interesting feature in Fig. 4 are higher concentrations of ASAS RRab stars at certain values of period and amplitude. We do not know whether this is simply an observational effect or RRab stars indeed prefer certain values of period and amplitude and are likely to adopt them. However, these concentrations are present in density maps plotted for limited subsamples of RRab stars which suggests this effect is real.

For further analysis we separate Oosterhoff I and II groups with a dashed line in Fig. 4, described by an equation:

$$
\mathrm{Amp}_{\mathrm{V}}=-8.0 \times \log P-0.85
$$

\subsection{The Period-Color Diagram}

We plot ASAS fundamental mode RR Lyr stars on a period-color diagram in Fig. 5. We use two $V-I$ color values: at maximum light (left panel) and at minimum light (right panel). There is no evident dependence of $V-I$ color on period at minimum light but it appears at maximum light as was already observed for MACHO RRab stars by Kanbur and Fernando (2005) and is explained by interaction between the photosphere and ionization front. In addition, our plots show a separation into Oosterhoff groups visible only at maximum light, at minimum light both groups are mixed. Oo I RRab stars are marked with blue open squares and Oo II with red filled circles. The groups were separated with the line described by Eq. (5).
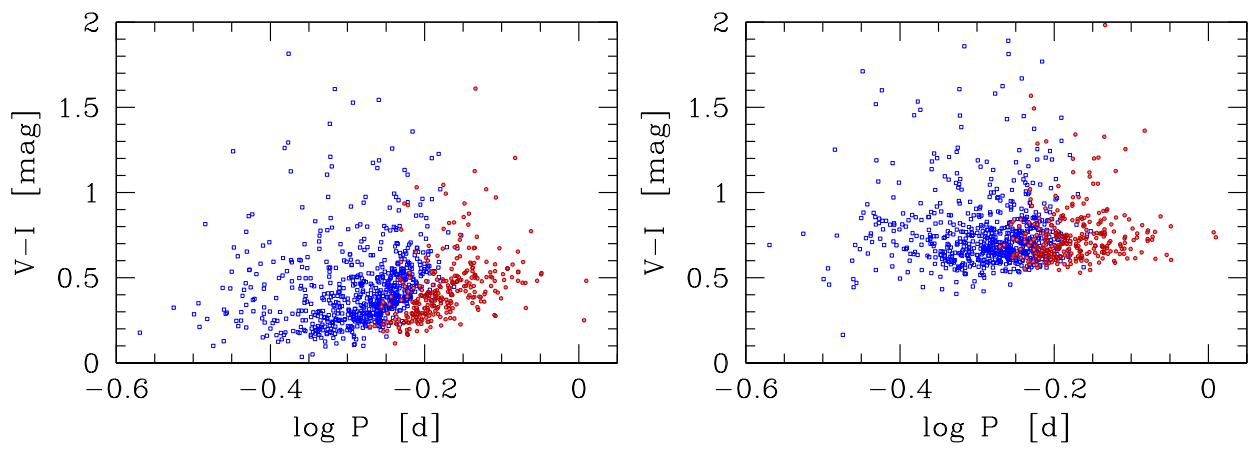

Fig. 5. Period-color diagram for ASAS RRab stars. Left panel presents $V-I$ color at maximum light, and the right panel at minimum light.

The data used in this investigation is available for download from the ASAS website. The form of the file is presented in Table 2. 
Table 2

ASAS RRab stars data

\begin{tabular}{|c|c|c|c|c|c|c|c|}
\hline ID & $\begin{array}{l}\text { RA } \\
{[\mathrm{h}]}\end{array}$ & $\begin{array}{l}\text { Dec } \\
{[\operatorname{deg}]}\end{array}$ & $\begin{array}{c}1 \\
{[\mathrm{deg}]}\end{array}$ & $\begin{array}{c}\mathrm{b} \\
{[\mathrm{deg}]}\end{array}$ & $\begin{array}{c}\mathrm{P} \\
\text { [days] }\end{array}$ & $\begin{array}{c}\mathrm{HJD}_{0}-2450000 \\
\text { [days] }\end{array}$ & \\
\hline $000248-2456.7$ & 0.046667 & -24.945 & 41.513967 & -78.863793 & 0.4933545 & 1870.54 & \\
\hline 000301-7041.5 & 0.050278 & -70.69167 & 308.649749 & -45.895328 & 0.5538941 & 1872.08 & \\
\hline $000321+0323.9$ & 0.055833 & 3.39833 & 100.269549 & -57.342245 & 0.5790441 & 1870.42 & \\
\hline $\begin{array}{c}V_{\min } \\
{[\mathrm{mag}]}\end{array}$ & $\begin{array}{l}V_{\max } \\
{[\mathrm{mag}]}\end{array}$ & $\begin{array}{c}V_{\text {avg }} \\
{[\mathrm{mag}]}\end{array}$ & $\begin{array}{l}\mathrm{Amp}_{V} \\
{[\mathrm{mag}]}\end{array}$ & $\begin{array}{c}I_{\min } \\
{[\mathrm{mag}]}\end{array}$ & $\begin{array}{c}I_{\max } \\
{[\mathrm{mag}]}\end{array}$ & $\begin{array}{c}I_{\mathrm{avg}} \\
{[\mathrm{mag}]}\end{array}$ & $\begin{array}{l}\mathrm{Amp}_{I} \\
{[\mathrm{mag}]}\end{array}$ \\
\hline 10.749 & 9.543 & 10.307 & 1.248 & 10.161 & 9.358 & 9.820 & 0.833 \\
\hline 14.034 & 13.139 & 13.683 & 0.877 & 13.392 & 12.846 & 13.190 & 0.572 \\
\hline 13.924 & 13.174 & 13.612 & 0.842 & 13.375 & 12.828 & 13.069 & 0.640 \\
\hline
\end{tabular}

The file contains 15 columns described in the header of the table and its full version is available for download from the ASAS website.

\section{Metallicity Determination}

In the era of photometric sky surveys, the number of spectroscopic observations from which metallicities are usually extracted is a few orders of magnitude lower than the amount of photometric observations. Fortunately for fundamental mode RR Lyr variables photometry itself can be used to calculate their metallicities with satisfactory accuracy. There are two widely used methods of metallicity determination for RR Lyr stars using their light curves: one by Jurcsik and Kovács (1996) and one by Sandage (2004). We will refer to them as JK96 and S04.

\subsection{Methods of Metallicity Determination}

In the method of JK96 metallicity is calculated from the period of the star $P$ and the phase combination $\varphi_{31}=\varphi_{3}-3 \varphi_{1}$, where $\varphi_{3}$ and $\varphi_{1}$ phases come from the Fourier sine decomposition of the $V$-band light curve (see Eq. 1):

$$
[\mathrm{Fe} / \mathrm{H}]_{\mathrm{JK}}=-5.038-5.394 P+1.345 \varphi_{31} \text {. }
$$

S04 transformed $[\mathrm{Fe} / \mathrm{H}]_{\mathrm{JK}}$ to the Zinn and West (1984) system that will be used in this paper:

$$
[\mathrm{Fe} / \mathrm{H}]_{\mathrm{JKZW}}=1.05 \times[\mathrm{Fe} / \mathrm{H}]_{\mathrm{JK}}-0.20 .
$$

This method was already successfully applied to a small sample of ASAS RRab stars by Kovács (2005). In Fig. 6 we compare metallicities calculated by us (details will follow in the next section) with the ones computed by Kovács (2005) for a set of ASAS light curves. The whole sample consists of 101 variables, and 
the filled circles (28 stars) represent a subsample that was also present in the paper of JK96. The average difference between metallicity values is $\left\langle[\mathrm{Fe} / \mathrm{H}]_{\text {diff }}\right\rangle=$ $\left\langle[\mathrm{Fe} / \mathrm{H}]_{\mathrm{JK}}-[\mathrm{Fe} / \mathrm{H}]_{\mathrm{Kov}}\right\rangle=0.02$ dex and the scatter is $\sigma_{[\mathrm{Fe} / \mathrm{H}]_{\text {diff }}}=0.29$ dex. The number of objects falling in the \pm 0.3 dex region (marked with dotted lines) is 73 which constitutes $72 \%$. Given that metallicity values were obtained by means of the same method and applied to almost the same light curves, we would expect to obtain much more consistent results. However, light curves in the sample of Kovács contain about $30 \%$ less points than our sample and this may be the source of the discrepancy. Nevertheless, the inconsistency is still disturbing. For now we can assume that the 0.29 dex scatter is what we can expect from the Fourier decomposition procedure uncertainties. Later in the paper we will assume that 0.3 dex difference in metallicities is an intrinsic JK96 method error.

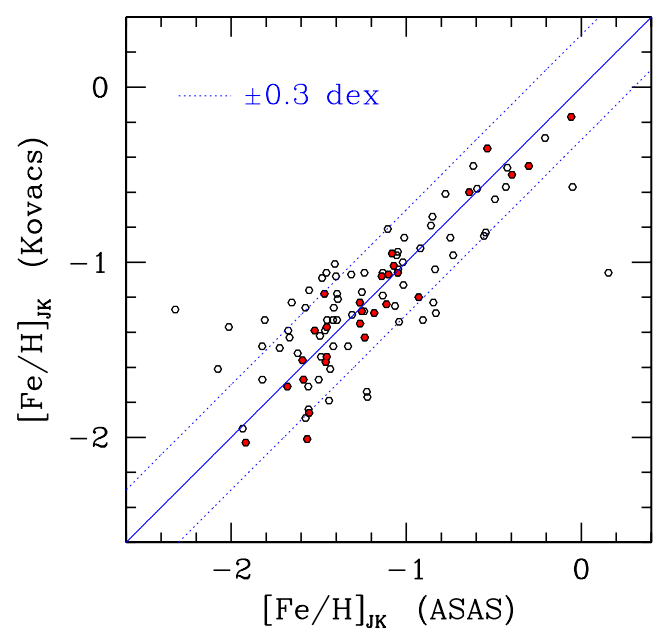

Fig. 6. Comparison of $[\mathrm{Fe} / \mathrm{H}]$ values from Kovács (2005) and this paper. Both were computed with the method of JK96 (Eq. 6) for a set of 101 ASAS light curves. Filled circles (28 stars) represent a subsample that was also present in the paper of JK96. The average difference between the two determinations is 0.02 dex and the scatter is 0.29 . The dotted lines delimit the \pm 0.3 dex region.

The method of S04 employs star's period and $V$-band amplitude:

$$
[\mathrm{Fe} / \mathrm{H}]_{\mathrm{S}}=-1.453 A_{V}-7.990 \log P-2.145
$$

and is less sensitive to light curve quality - it is always easier to determine accurately a total amplitude than phases of Fourier harmonics. However, an error of 0.1 mag in amplitude gives an error of almost 0.15 dex in metallicity. Such uncertainties usually occur for Blazhko RR Lyr stars, as the amplitudes averaged over several cycles are usually lower than for non Blazhko variables producing larger metallicity values.

We will compare these two methods in the next Sections. 


\subsection{Metallicity Calculation for the Sample of ASAS RRab Stars}

We compute photometric metallicities for the 1227 ASAS RRab stars using both the method of JK96 and S04 (Eqs. 7 and 8). We have already refined (and in some cases recalculated) pulsation periods supplied by the ACVS and we can assume that they are correct to very high accuracy. In order to estimate light curve parameters $A_{V}$ and $\varphi_{31}$ we decomposed the light curves into Fourier sine series, as described in the last paragraph of Section 2.2. The amplitude $A_{V}$ is defined as the difference between the maximum and minimum values of the Fourier model. In addition we calculated phase errors $\varphi_{31, \text { err }}$ and we will consider them as an uncertainty measure in metallicity determination.

We tried to apply a compatibility test used by JK96 to verify whether a light curve had a good shape, i.e., was in agreement with their "basic sample" of the light curves. However, most of our light curves did not pass this test, having a maximum deviation parameter $D_{\max }$ much higher than the acceptable value 3.0. There were cases when calculated metallicity was in good agreement with the spectroscopic value and the light curve was of high quality but the deviation parameter was very high. For this reason and to avoid detailed investigation of each of 1227 light curves we used $\varphi_{31, \text { err }}$ alone as a measure of metallicity error, as we can ignore the error in period estimation.
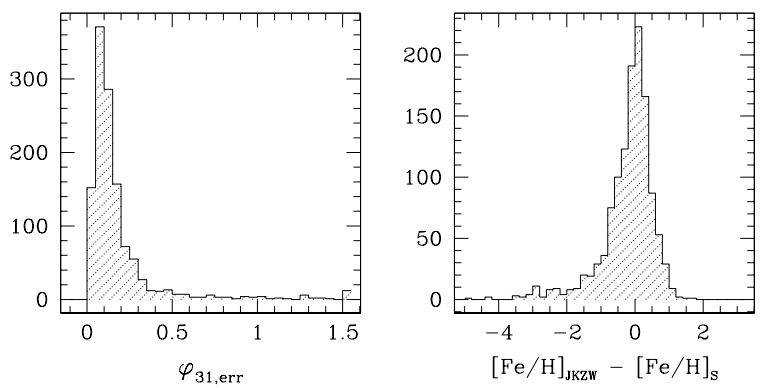

Fig. 7. Left panel shows distribution of phase errors $\varphi_{31 \text {,err }}$ and the right panel the distribution of differences between metallicities calculated from method of JK96 and S04. The majority of objects have well defined $\varphi_{31}$ values (below 0.3 ) but nevertheless the metallicity differences are significant.

Fig. 7 presents a histogram of $\varphi_{31, \text { err }}$ (left panel) and a histogram of differences of metallicities calculated with two methods $[\mathrm{Fe} / \mathrm{H}]_{\text {diff }}=[\mathrm{Fe} / \mathrm{H}]_{\mathrm{JKZW}}-[\mathrm{Fe} / \mathrm{H}]_{\mathrm{S}}$ (right panel). The average difference is $\left\langle[\mathrm{Fe} / \mathrm{H}]_{\text {diff }}\right\rangle=-0.17$ dex with the majority of the outliers located at $[\mathrm{Fe} / \mathrm{H}]_{\text {diff }}<-2.0$ dex. When we discard objects with $\left|[\mathrm{Fe} / \mathrm{H}]_{\text {diff }}\right|>1.5$ dex the average is $\left\langle[\mathrm{Fe} / \mathrm{H}]_{\text {diff }}\right\rangle=-0.04$. The discrepancy between methods is not unexpected, as most stars in the sample are faint and have noisy light curves and both $\varphi_{31 \text {,err }}$ and the scatter of $[\mathrm{Fe} / \mathrm{H}]_{\text {diff }}$ increase with magnitude, as shown in Fig. 8.

After visual inspection of the light curves with large errors and metallicity differences, we decided to reject objects with $\varphi_{31, \text { err }}>0.3$ (131 stars) and with 

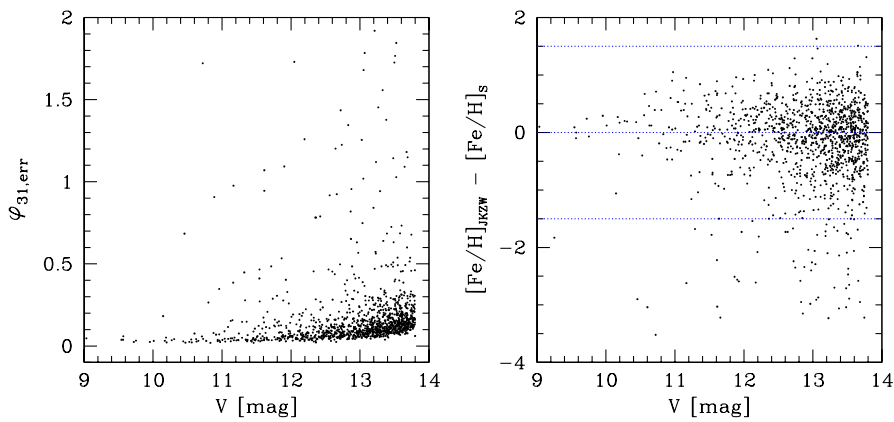

Fig. 8. Photometric metallicity estimation dependence on star magnitudes - the fainter the star the larger is the phase error (left panel), and similarly - the fainter the star, the larger is the discrepancy between metallicities calculated with different methods (right panel).

$\left|[\mathrm{Fe} / \mathrm{H}]_{\text {diff }}\right|>1.5$ (73 stars) which altogether gives 155 objects. There still remain objects that have large metallicity differences, namely 52 RRab stars with $\left|[\mathrm{Fe} / \mathrm{H}]_{\text {diff }}\right|>1$. Some of them have quite noisy light curves and we could accept this as a satisfactory explanation but some objects have well defined light curves and both amplitude and $\varphi_{31}$ phase combination possess small errors, for example ASAS 135141+0625.9 and 221635-0349.0 pictured in Fig. 9. We decided to leave all these stars in the sample for further analysis. In 43 (out of 52) such cases $[\mathrm{Fe} / \mathrm{H}]_{\mathrm{JKZW}}$ is smaller than $[\mathrm{Fe} / \mathrm{H}]_{\mathrm{S}}$.
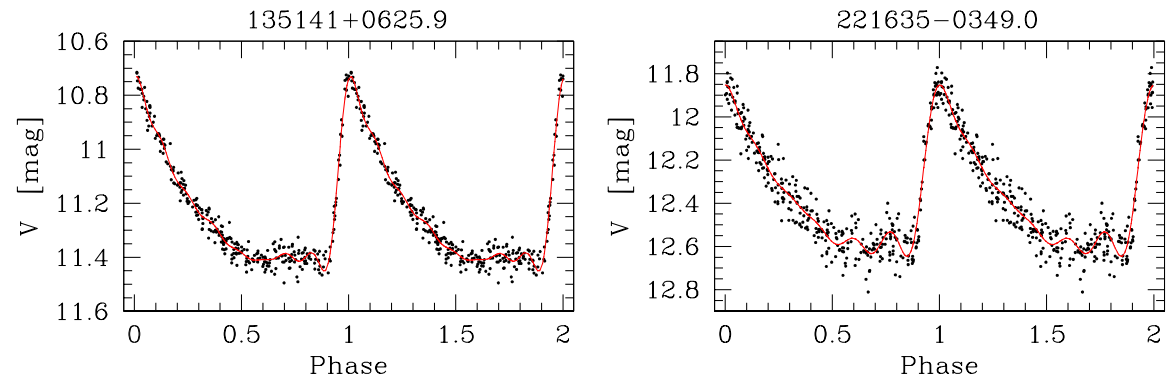

Fig. 9. Two examples of well defined RRab stars light curves showing large differences in photometric metallicity determination. For ASAS $135141+0625.9$ (left panel) $[\mathrm{Fe} / \mathrm{H}]_{\mathrm{JKZW}}=-1.74$ and $[\mathrm{Fe} / \mathrm{H}]_{\mathrm{S}}=-0.55$ and for ASAS 221635-0349.0 (right panel) $[\mathrm{Fe} / \mathrm{H}]_{\mathrm{JKZW}}=-4.63$ and $[\mathrm{Fe} / \mathrm{H}]_{\mathrm{S}}=-3.37$. The line is a 6th order Fourier fit to the light curve.

Szczygieł and Fabrycky (2007) identified 180 Blazhko or double mode RR Lyr stars in the ASAS data. These light curves display a large scatter which strongly affects amplitude and Fourier parameters determination and would change their true photometric metallicities. However, Smolec (2005) showed that after prewhitening the data with additional close frequencies reliable photometric metallicity values can be obtained. Given that the check was performed only on five well observed stars from a narrow metallicity range, we decide to exclude all multiperiodic RRab 
stars from the final sample.

\subsection{Comparison of Photometric Metallicities from Two Methods}

Now we compare $[\mathrm{Fe} / \mathrm{H}]_{\mathrm{JKZW}}$ and $[\mathrm{Fe} / \mathrm{H}]_{\mathrm{S}}$ metallicities of the remaining 1008 RRab stars by plotting the two values against each other in the left part of Fig. 10 and taking into account Oosterhoff type as assigned in Section 3.1. Oosterhoff I type RRab stars are marked with blue squares and Oosterhoff II with red circles. While there is a rough agreement between methods, we see two distinct concentrations of points which are apparently a result of Oosterhoff dichotomy. As previously shown in Fig. 8, the largest errors in metallicity estimation and the largest differences between methods are at fainter magnitudes. However, plotting a subsample of variables brighter than $V=12.5$ mag ( 255 objects) does not change the overall structure of the image.

The distributions of $[\mathrm{Fe} / \mathrm{H}]_{\mathrm{JKZW}}$ and $[\mathrm{Fe} / \mathrm{H}]_{\mathrm{S}}$ are presented in the right part of Fig. 10. There is a clear bimodal distribution of S04 metallicities, while the JK96 distribution is uniform. In the case of S04 method a separation into Oosterhoff groups is responsible for the bimodality: almost all Oo I RRab stars have metallicities larger than -1.75 dex while Oo II smaller than -1.6 dex. This is not observed in the case of JK96 method, where Oo I type RRab stars adopt metallicities from the wide range starting at -2.5 dex, while Oo II RRab stars stay in the low metallicity regime below -1.0 dex. Average metallicity values differ significantly between methods and in the case of S04 method are -2.06 dex for Oo II and -1.12 dex for Oo I and in the case of JK96 -1.85 dex for Oo II and -1.27 dex for Oo I. This sharp division in the case of S04 method is not physical and is not in agreement with metallicity distribution of spectroscopically observed ASAS RRab stars, as described in the following section.

Another observation from Fig. 10 is that the most extreme differences in $[\mathrm{Fe} / \mathrm{H}]$ at very low and very high metallicities have larger $[\mathrm{Fe} / \mathrm{H}]_{S}$ values, which again suggests that one of the methods does not work well at untypical metallicities.

\subsection{Comparison with Spectroscopic Metallicities}

We combine spectroscopically obtained metallicities published by Layden (1994) with photometric metallicities calculated for the ASAS RRab stars sample. The combination results in 186 objects and is presented in Fig. 11. Left panel shows the comparison with method of JK96 and the middle panel with S04. The dotted lines on both images delimit the \pm 0.3 dex region. It is clear that the method of S04 produces larger scatter than the method of JK96 and two concentrations described in the previous section are present in the middle panel, but poorly visible.

We perform a star to star comparison for both sets in order to see whether the method of JK96 is significantly better than S04. The average difference between photometric and spectroscopic metallicity values in case of JK96 method is 0.03 dex and the dispersion is 0.30 dex. $75 \%$ stars lie within the \pm 0.3 dex lines. In case 

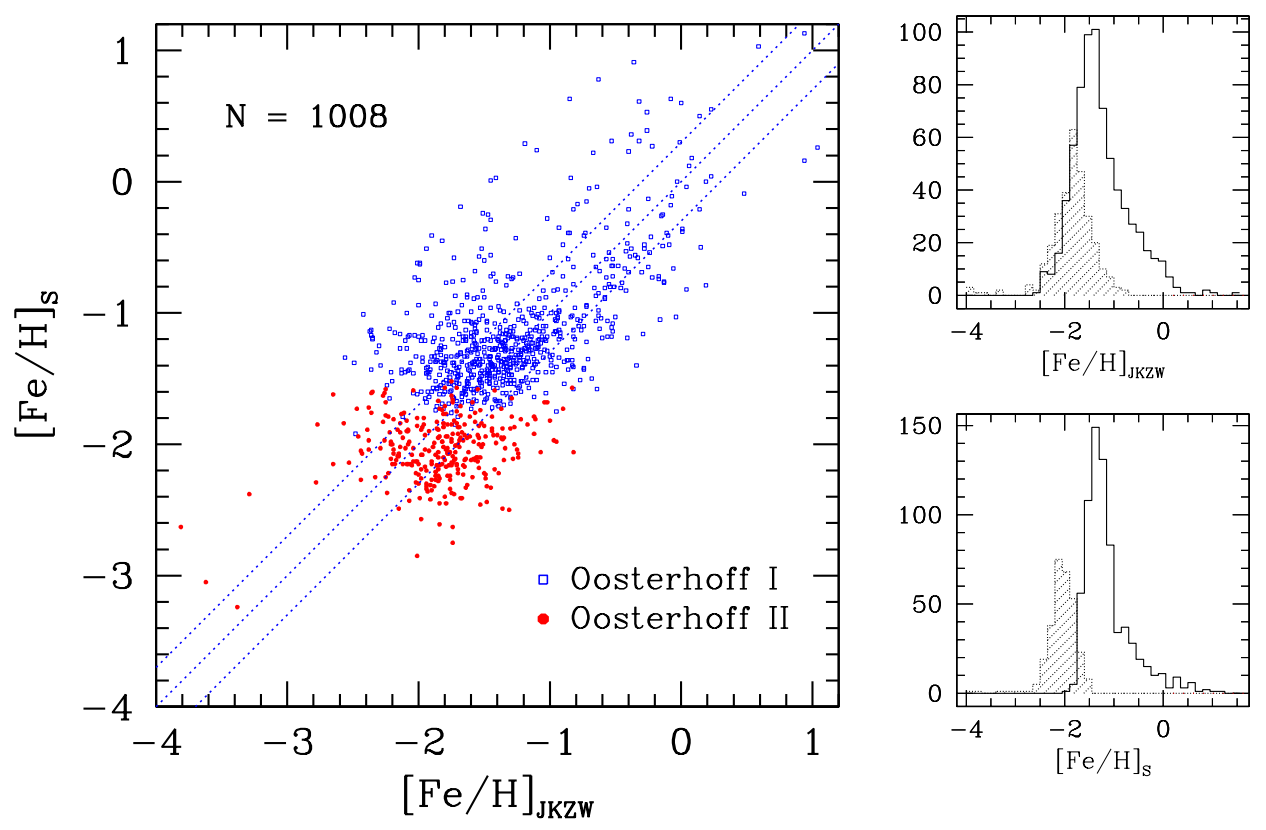

Fig. 10. Comparison of two methods of photometric metallicity estimation is presented on the left plot. $[\mathrm{Fe} / \mathrm{H}]_{\text {JKZW }}$ is computed by the method of Jurcsik and Kovács (1996) and $[\mathrm{Fe} / \mathrm{H}]_{\mathrm{S}}$ by that of Sandage (2004). Oosterhoff groups are marked with different symbols: Oo I with blue squares and Oo II with red circles. The three dotted lines are separated by 0.3 dex. Two distinct concentrations of points are visible and are apparently a result of Oosterhoff dichotomy. Two histograms on the right present distribution of photometric metallicities from JK96 (top panel) and S04 (bottom panel) methods. Shaded areas on the histograms correspond to Oo II group (296 stars) and the clear ones to Oo I group (712 stars). While the distribution of $[\mathrm{Fe} / \mathrm{H}]_{\text {JKZW }}$ is uniform, the $[\mathrm{Fe} / \mathrm{H}]_{\mathrm{S}}$ metallicity distribution is bimodal and corresponds to the Oosterhoff separation. The mean metallicities are $\left\langle[\mathrm{Fe} / \mathrm{H}]_{\mathrm{JKZW}}\right\rangle=-1.85$ dex for Oo II and $\left\langle[\mathrm{Fe} / \mathrm{H}]_{\mathrm{JKZW}}\right\rangle=-1.27$ dex for Oo I (top panel), and $\left\langle[\mathrm{Fe} / \mathrm{H}]_{\mathrm{S}}\right\rangle=-2.06$ dex for Oo II and $\left\langle[\mathrm{Fe} / \mathrm{H}]_{\mathrm{S}}\right\rangle=-1.12$ dex for Oo I.
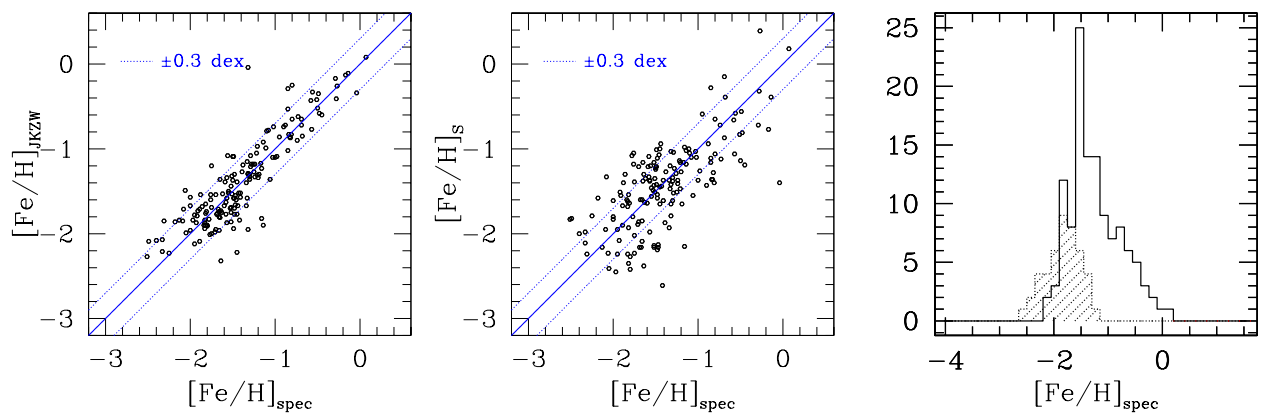

Fig. 11. Comparison of spectroscopically determined metallicity values from Layden (1994) with photometrically calculated ones using the method of JK96 (left panel) and S04 (middle panel), for the sample of 186 RRab stars. The dotted lines on both images delimit the \pm 0.3 dex region. The right panel presents a distribution of spectroscopic metallicities for ASAS RRab stars - the shaded area corresponds to Oo II group and the clear area to Oo I group. 
of S04 method the difference is 0.02 dex and the scatter is 0.43 dex. Only $50 \%$ stars fall within \pm 0.3 dex region. Summarizing, JK96 method is better calibrated against spectroscopic metallicities and the conformity holds reasonably well within the whole metallicity range. Photometric metallicities from S04 method seem to be underestimated in low metallicity range and overestimated at high metallicity range, suggesting that there should be separate formulae for the two Oosterhoff groups.

Finally, right panel of Fig. 11 presents spectroscopically obtained metallicity distribution of ASAS RRab stars, with distinguished Oosterhoff groups - the shaded area corresponds to Oo II group and the solid line defines the area of Oo I group. Similarly to the histogram of photometric JK96 metallicities (Fig. 10), Oo I RRab stars cover metallicity values from a wide range between -2.2 and 0.2 dex, overlapping with metallicities typical for Oo II group, between -2.6 and -1 dex. This disagrees with a sharp separation of photometric metallicity values of S04 among Oosterhoff groups, visible on the bottom histogram of Fig. 10.
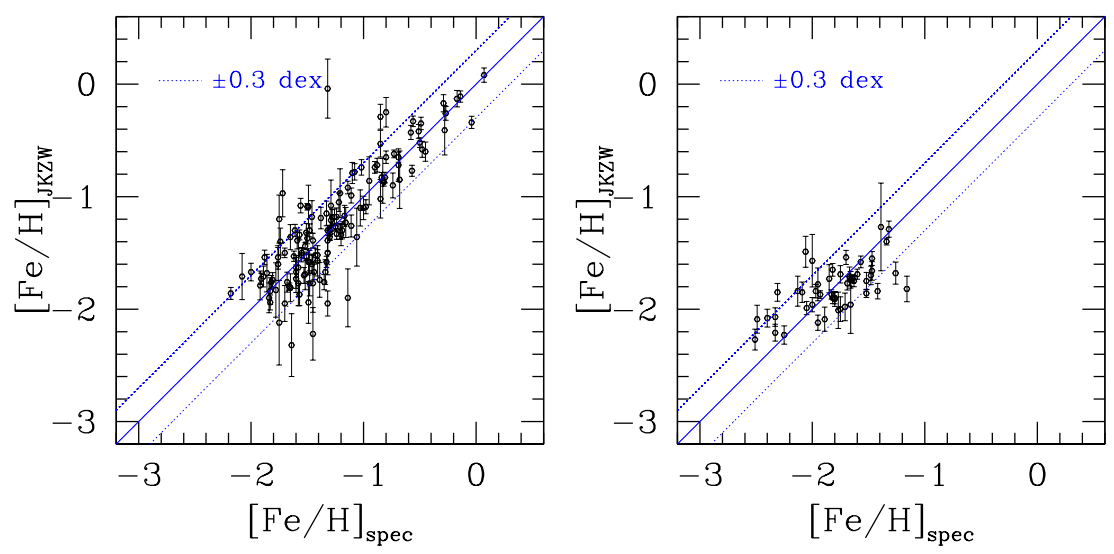

Fig. 12. Comparison of spectroscopically determined metallicity values from Layden (1994) with photometrically calculated ones using the method of JK96. Left panel presents Oosterhoff I RRab stars and the right panel Oosterhoff II RRab stars. The dotted lines on both images delimit the \pm 0.3 dex region.

Although the method of JK96 produces metallicities in good agreement with spectroscopic ones, there is an increased scatter in the lower part of the left plot in Fig. 11, for $[\mathrm{Fe} / \mathrm{H}]_{\mathrm{JKZW}}<1.5$ dex. We plot a comparison of JK96 metallicities with spectroscopic ones again (Fig. 12), but this time separately for each Oosterhoff group (Oo I in the left panel and Oo II in the right). It is visible that most of the scatter is introduced by the Oo II group, for which a correlation is not as tight as for Oo I variables. There are also several outliers in the low metallicity range in case of Oo I type RRab stars, but these measurements have high $[\mathrm{Fe} / \mathrm{H}]_{\mathrm{JKZW}}$ errors, while the errors of the outliers in Oo II group are small. This would imply that separate metallicity estimation formulae should be constructed for different Oosterhoff groups, not only for S04 method, but also for JK96. We think this is 
conceivable, given that Oosterhoff I and II type RRab stars are of different age, Oo II being more advanced in their evolution. It is possible that their pulsation has changed producing slightly different light curve shape, which would affect metallicity dependence on Fourier parameters of the light curve decomposition. We tried to modify the existing formula (Eq. 6), with no satisfactory results - the obtained relations were very similar to the present one and did not reduce the scatter when compared with spectroscopic metallicities. It looks as a linear dependence of the metallicity on period and phase parameter is not sufficient to describe this relation and other components might have to be included. In any event, more spectroscopic observations of RRab stars with small metal content would be desirable.

\subsection{Final Metallicity Values}

In previous subsections we presented two methods of photometric metallicity determination and compared them with each other showing, that there is a significant discrepancy especially in two regions of metallicities. Comparison of both methods with spectroscopic results undoubtedly favors the JK96 method, which shows better conformity and smaller scatter and is less sensitive to Oosterhoff type. For this reason we will use JK96 metallicities only.

Ta b le 3

Metallicity values of selected ASAS RRab stars

\begin{tabular}{ccccccc}
\hline ID & {$[\mathrm{Fe} / \mathrm{H}]_{\mathrm{JKZW}}$} & {$[\mathrm{Fe} / \mathrm{H}]_{\mathrm{JKZW}, \text { err }}$} & {$[\mathrm{Fe} / \mathrm{H}]_{\mathrm{S}}[\mathrm{Fe} / \mathrm{H}]_{\mathrm{NSVS}}[\mathrm{Fe} / \mathrm{H}]_{\text {spec }}$} & {$[\mathrm{Fe} / \mathrm{H}]_{\mathrm{spec}, \text { err }}$} \\
\hline $000248-2456.7$ & -1.25 & 0.028 & -1.44 & - & -1.25 & 0.130 \\
$000301-7041.5$ & -1.35 & 0.230 & -1.41 & - & - & - \\
$000321+0323.9$ & -1.86 & 0.281 & -1.36 & -1.69 & - & - \\
\hline
\end{tabular}

The first column contains ASAS ID. The following columns contain metallicity values: from the JK96 method and its error, from the S04 method, for the NSVS counterpart calculated by Kinemuchi et al. (2006), and finally spectroscopic value from Layden (1994) and its error. The full version of this Table is available for download from the ASAS website.

Kinemuchi et al. (2006) also determined photometric metallicities for their 589 fundamental mode RR Lyr stars, using both methods. For 363 stars that had sufficient number of observations and a good phase coverage, they took an average from both methods as their metallicity value. For the remaining 226 objects they applied the metallicity of S04 alone, assuming it is more reliable in case of lower quality light curves. However, as shown in the Appendix, NSVS amplitudes are not always well determined and the S04 method itself is not reliable enough, thus we expect a majority of NSVS metallicity values to be highly uncertain. We compared ASAS and NSVS metallicity values for stars from the overlapping region and concluded, that the mean absolute difference in metallicities is fairly high, around $0.3 \mathrm{dex}$. The details of this comparison are presented in the Appendix. This is a serious argument for not combining both samples to investigate metallicity based properties of 
all Galactic RRab stars from both hemispheres.

Calculated metallicities are available for download from the ASAS website. The exemplary lines of the file are presented in Table 3.

\section{Galactic Distribution}

It would be interesting to combine ASAS and NSVS RR Lyr stars catalogs in order to investigate Galactic distribution of the complete RRab stars sample in the Solar neighborhood and we initially intended to do so. The data from both projects were obtained with similar equipment and cover very similar magnitude range, but telescopes observed from different hemispheres thus providing a complete sky coverage of the whole sphere. Unfortunately, when comparing the ASAS and NSVS data in the overlapping region we encountered several inconsistencies concerning RR Lyr stars detection efficiency in both catalogs - among 967 ASAS objects and 806 NSVS objects in this area only 446 are common in the whole magnitude range. When we narrow the magnitude range to 10-12 mag (in which both projects should have complete samples) these numbers are 114 for ASAS and 72 for NSVS, with 63 common stars, showing that the catalogs are neither complete nor compatible. The more detailed comparison of the catalogs is presented in the Appendix. Such inconsistency excludes any statistical conclusions based on RR Lyr stars absolute numbers.

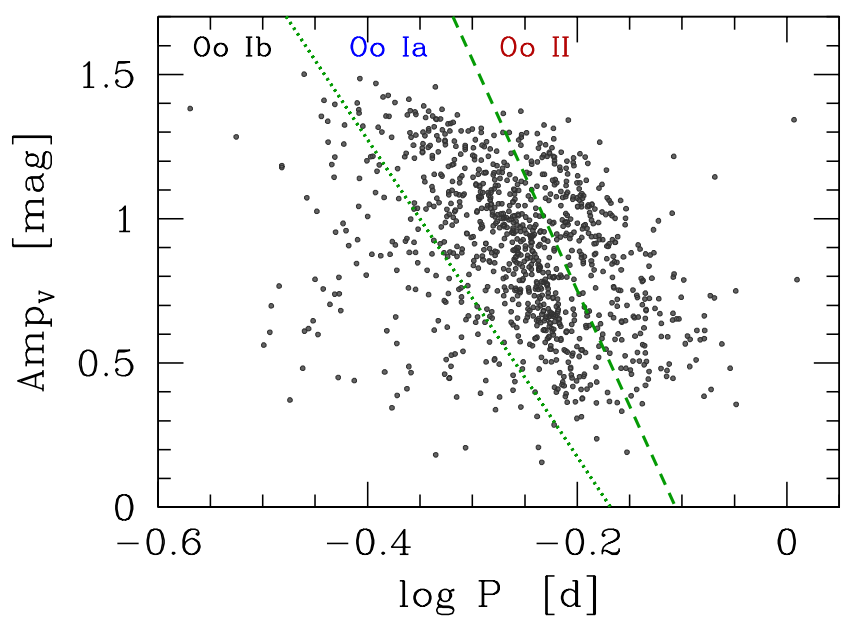

Fig. 13. Period-amplitude (Bailey) diagram for ASAS RRab stars. Lines divide points into three groups: right side of the dashed line contains points from Oosterhoff II group, the points between dashed and doted lines (being the main part of Oosterhoff I group) are assigned to Oosterhoff Ia group while the remaining points to the left side of the dotted line are the Oosterhoff Ib group.

Additional difficulties were met when comparing photometric metallicities, as described in the previous section. A mean absolute difference in metallicities of $\approx$ 
0.3 dex induces a difference in calculated distance $\approx 130 \mathrm{pc}$ for $V=12$ magnitude star and $\approx 340 \mathrm{pc}$ for $V=14$ magnitude star. This is another argument against combining both databases and investigating total distance distributions or density functions.

These differences, preventing from combining both catalogs, at the same time encouraged us to inspect distribution of ASAS RRab variables and to compare it to results of Kinemuchi et al. (2006) for NSVS data. For the following analysis we divided ASAS RRab stars into three groups, as pictured in Fig. 13. The Oosterhoff II group remains unchanged and consists of all points to the right of the dashed line given by Eq. (5). Similarly to Kinemuchi et al. (2006), we have plotted in Fig. 13 the dotted line corresponding to S04 constant metallicity line of $[\mathrm{Fe} / \mathrm{H}]=-0.8$.

This line splits Oosterhoff I group into two subgroups that we will call Oo Ia (numerous, longer period, lower metallicity stars located between dotted and dashed lines), and Oo Ib (sparse, shorter period, higher metallicity stars located on the left side of the dotted line).

\subsection{Distance Determination}

Distances to ASAS RRab stars were determined using the distance modulus, without correction for extinction. We assume that at short distances such as exhibited by ASAS RR Lyr stars extinction does not play a significant role. The distance $d$ from the Sun is calculated as follows:

$$
d=10^{0.2\left(m_{V}-M_{V}+5\right)}
$$

where $m_{V}$ and $M_{V}$ are apparent and absolute average magnitudes in $V$-band, respectively. Absolute magnitudes $M_{V}$ were calculated using formula from Bono, Caputo and Di Criscienzo (2007):

$$
M_{V}=1.19+0.5[\mathrm{Fe} / \mathrm{H}]_{\mathrm{JKZW}}+0.09[\mathrm{Fe} / \mathrm{H}]_{\mathrm{JKZW}}^{2} .
$$
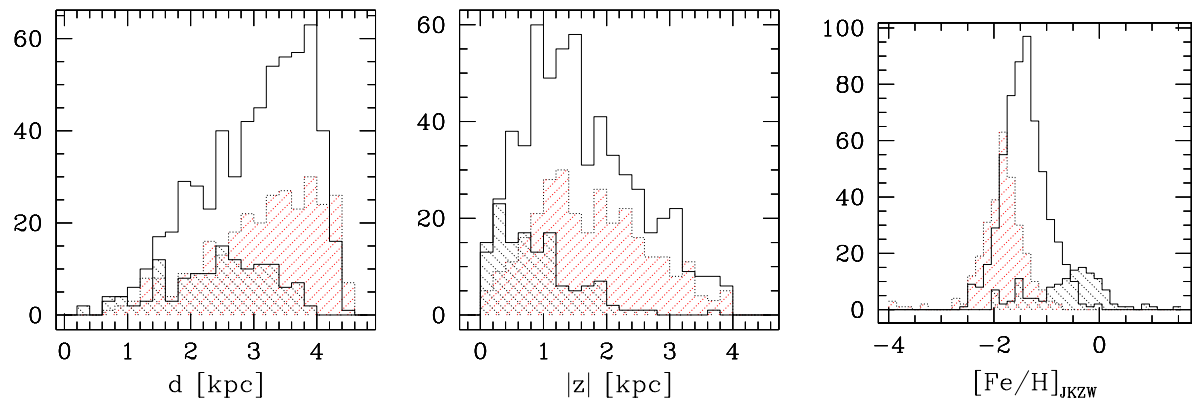

Fig. 14. The distributions of distance $d$ from Sun (left panel) and distance $|z|$ from the Galactic plane (middle panel) of ASAS RRab stars. Right panel presents metallicity distributions. On all three histograms plain area corresponds to Oo Ia group, the red shaded area to Oo II group, and the black shaded area to $\mathrm{Oo} \mathrm{Ib}$ group. 
The distribution of distances $d$ from the Sun is presented in the left panel of Fig. 14. If we assume that ASAS is complete to $V=13.5 \mathrm{mag}$, then the distance to which we should observe all RRab stars is about $4 \mathrm{kpc}$. This is in agreement with distance distributions for Oo II and Oo Ia groups - the maximum of Oo Ia and Oo II histograms is at $4 \mathrm{kpc}$ and detection falls rapidly for larger distances. However, in the case of Oo Ib stars (most metal rich RRab stars, as seen in the right panel of Fig. 14) the maximum of the histogram is at $2.5 \mathrm{kpc}$. At the same time this group of metal rich stars is concentrated close to the Galactic plane (middle panel of Fig. 14), with the majority of stars having $|z|<1 \mathrm{kpc}$. These two properties coincide with the location of the spiral arms of the Milky Way at a distance approximately $2 \mathrm{kpc}$ (Xu et al. 2006) and it would be tempting to locate Oo Ib RRab stars there. However, we must remember that distances were calculated without correction for extinction which is not negligible at low galactic latitudes, thus their errors may be significant. The middle panel of Fig. 14 also shows that both Oosterhoff I and II groups are present at all values of $|z|$, that is, the local neighborhood is a mixture of older (metal poor) and younger (metal rich) stars, whereas the youngest stars are found only at low values of $|z|$, that is in the thick disk region.
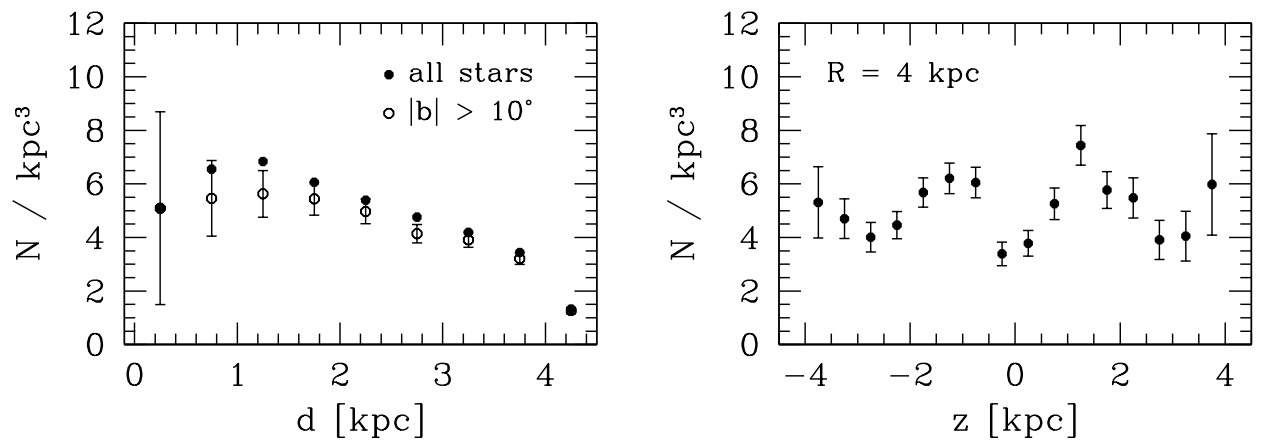

Fig. 15. Densities of ASAS RRab stars presented as a number of stars per kiloparsec cube. The left panel presents the density change with distance $d$ from the Sun and the filled circles represent a whole sample of 1008 RRab stars, while the open circles a subsample of 922 RRab stars with absolute galactic latitude greater than $10^{\circ}$. The right panel shows the density as a function of distance $z$ from the Galactic plane. The error bars were calculated assuming the Poissonian distribution of star numbers in each bin. For details on the density calculation see text.

In addition, we calculated densities of ASAS RRab stars depending on the distance $d$ from the Sun and from the Galactic plane $z$ and the results are presented in Fig. 15. In case of the distance from the Sun (left panel of Fig. 15) the distance bin used to calculate the number of stars per kiloparsec was set to $500 \mathrm{pc}$, so the densities were calculated for objects within concentric spheres with the difference in radius equal to $500 \mathrm{pc}$. The calculations were performed for two data sets: one contained all 1008 ASAS RRab stars for which the distances were calculated (filled symbols in the left panel of Fig. 15) and the other contained objects for which the 
absolute galactic latitude was greater than $10^{\circ}$ (open symbols). As expected, the density is fairly constant within the Sun's proximity and the slope of the decrease is small. In case of the distance $z$ from the galactic plane (right panel of Fig. 15) the densities were calculated within the slices of the $4 \mathrm{kpc}$ radius sphere, and the thickness of a slice was set to $500 \mathrm{pc}$. The density fluctuations are higher due to smaller number of stars per bin, but the density stays roughly constant in higher $z$ regions within the error bars. With decreasing $z$ an increase in density is observed, as expected, but it drops in the low region of $z$ in which the ASAS resolution fails. When calculating the densities described above the correction for a limited ASAS vision was included. The error bars in Fig. 15 were calculated assuming the Poissonian distribution of star numbers in each bin.

\subsection{Metallicity Distribution in the Galaxy}

Kinemuchi et al. (2006) noticed that metal rich stars (with $[\mathrm{Fe} / \mathrm{H}]>-1$ ) are associated with the Galactic disk and confirmed this conclusion by calculating their scale heights. As shown in the previous Section (Fig. 14), our Oo Ib sample is evidently concentrated toward the Galactic plane, but it does not contain all metal rich RRab stars. In Fig. 16 we plot metallicity values of all 1008 ASAS RRab stars against absolute distance from the Galactic plane $|z|$. Here it is clear that almost all metal rich RRab stars $([\mathrm{Fe} / \mathrm{H}]>-1$ dex $)$ are located at low $|z|(<2 \mathrm{kpc})$ and they belong both to Oo Ia and Oo Ib groups. There is no clear separation line between the Oosterhoff types like the one visible in Fig. 14 of Kinemuchi et al. (2006) at $[\mathrm{Fe} / \mathrm{H}]=-1$ because we did not separate Oo Ia and Oo Ib groups with a constant $[\mathrm{Fe} / \mathrm{H}]$ line, mostly a result of using JK96 metallicity estimation method which does not have constant $[\mathrm{Fe} / \mathrm{H}]$ lines in $\log P$-Amp plane (for details refer to Section 4).

Within the main part of Oosterhoff I group (Oo I, blue squares) we notice a decrease in $|z|$ with increasing metallicity, which does not seem to be present among Oo II type stars. In the intermediate and low metallicity region, for $[\mathrm{Fe} / \mathrm{H}] \in$ $(-2.5,-1)$ dex the cloud is a mixture of both Oosterhoff I and II types, uniformly distributed at all values of $|z|$.

In Fig. 17 we plot metallicity values averaged in several distance bins in order to investigate global changes in metallicity across the Galaxy. The first row of images presents average metallicity values of all ASAS RRab stars against distance $d$ from the Sun (left panel), absolute distance $|z|$ from the Galactic plane (middle panel) and distance from the Galactic center $R$ (right panel). The distance of the Sun from the Galactic center was assumed to be $8 \mathrm{kpc}$. The second row presents analogous images, but metallicities are averaged within Oosterhoff groups - red filled circles stand for Oo II RRab stars, blue open squares for the main part of Oosterhoff I group, namely Oo Ia, and black crosses represent an Oo Ib subgroup.

The overall image emerging from Fig. 17 is consistent with the general model of the Galaxy - the closer to the Sun or to the Galactic plane we are, the higher are 


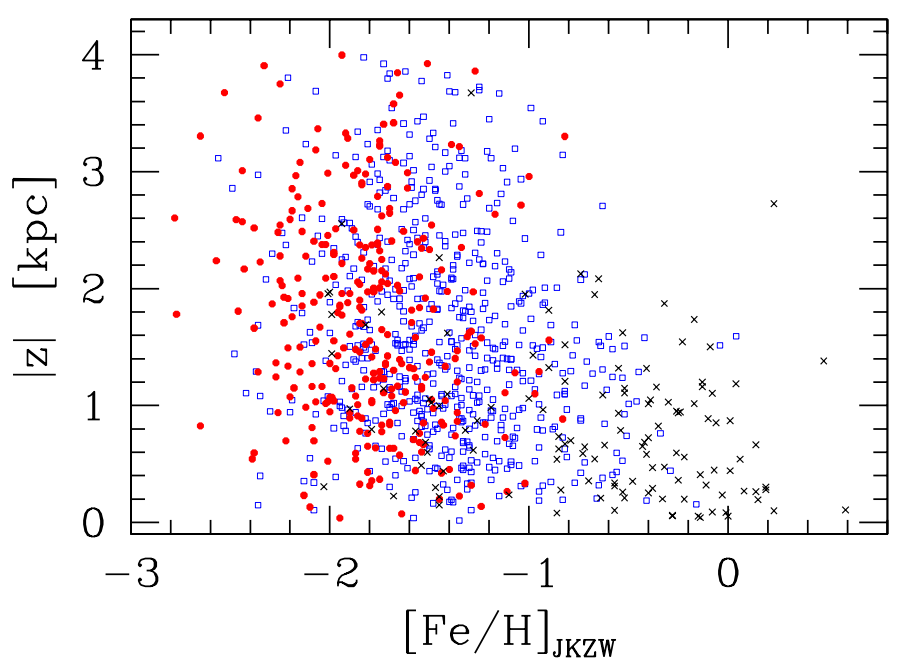

Fig. 16. The distribution of ASAS RRab stars metallicities against distance from the Galactic plane. Red filled circles represent Oosterhoff II group, blue open squares stand for the main part of the Oosterhoff I group (Oo Ia) while black crosses represent Oo Ib stars.

the metallicities of ASAS RRab stars. When we distinguish between Oosterhoff types it is clear that the largest contribution to this rise comes from the metal rich Oo Ib stars which mostly occur at lower $|z|$. In other words: younger, more metal rich stars (Oo I) are concentrated toward the disk, while the older, more metal poor stars (Oo II) are on average at larger distances from the Galactic plane. Both Oo Ia and $\mathrm{Oo} \mathrm{Ib}$ groups display higher metallicities when closer to the Sun, while the metallicity of Oo II remains fairly constant with $d$. The increase in metallicity with decreasing $|z|$ is present among all three Oo groups and becomes well defined at $|z| \approx 2.5 \mathrm{kpc}$. There is no apparent metallicity trend with distance from the Galactic center, as shown by Kinemuchi et al. (2006).

\section{Summary and Conclusions}

The aim of this paper was to analyze 1455 fundamental mode nearby RR Lyr stars using the All Sky Automated Survey data. In the process of data preparation we have inspected all $V$ and $I$-band RRab stars light curves, cleaned them and refined or recalculated their pulsation periods. During this stage we rejected stars that had low quality light curves.

We constructed a period-amplitude diagram for a reduced sample of stars and unlike in the previous analysis of Galactic field RRab stars (Kinemuchi et al. 2006) we noticed a clear presence of the Oosterhoff groups (Oosterhoff 1939) typical for globular cluster RR Lyr variables. This, together with the recent study of outer halo RR Lyr stars (Miceli et al. 2008) implies that the Oosterhoff dichotomy is present in the whole Milky Way Galaxy including a very close neighborhood of the Sun. It 

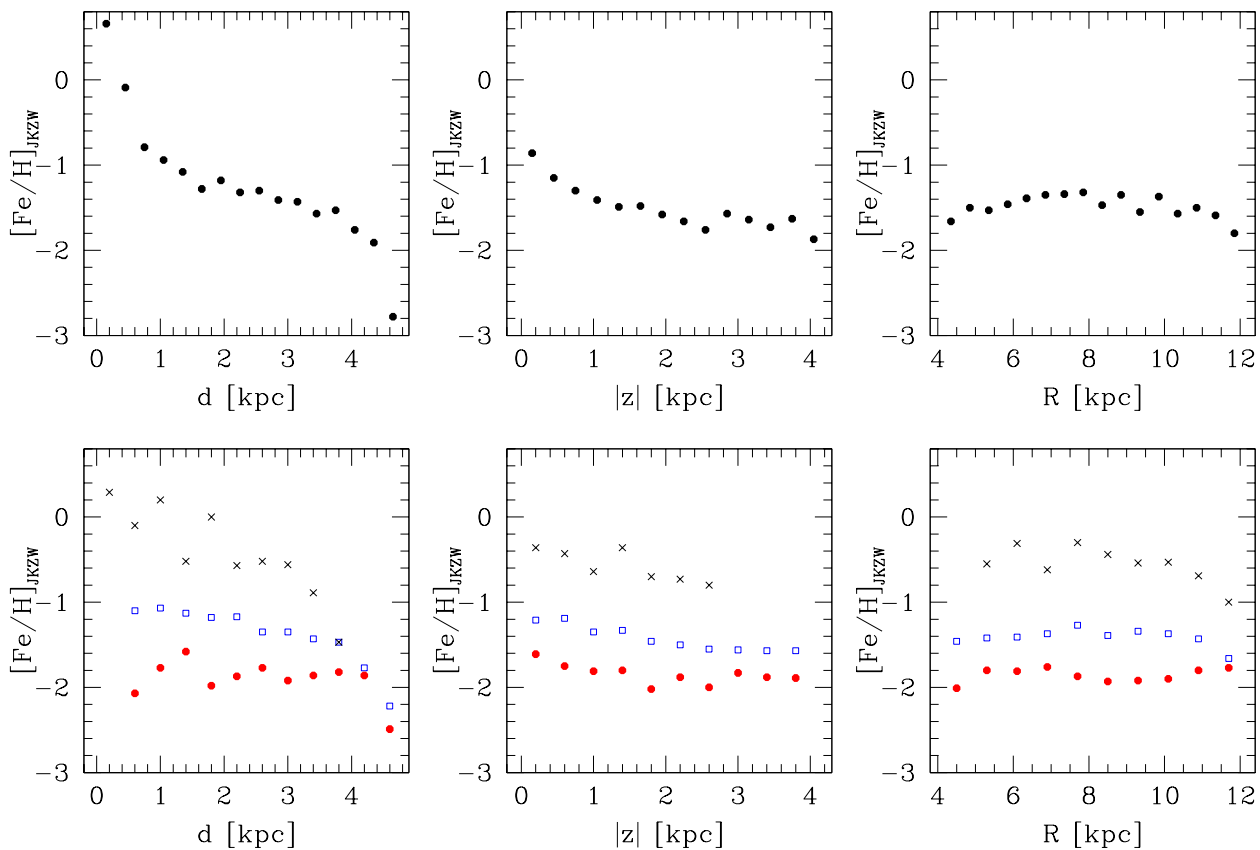

Fig. 17. All images present metallicity values averaged in distance bins. The first row plots show metallicity values of all ASAS RRab stars against distance $d$ from the Sun (left panel), absolute distance $|z|$ from the Galactic plane (middle panel) and distance from the Galactic center $R$ (right panel). The second row presents analogous images, but metallicities are averaged within Oosterhoff groups - filled circles stand for Oo II RRab stars, open squares for the main part of Oosterhoff I group, namely Oo Ia, and crosses represent an Oo Ib subgroup.

follows that the accretion of close-by dwarf galaxies as a scenario of early Galaxy formation can be excluded.

The $\log P-\mathrm{Amp}_{V}$ relation for Oosterhoff I RRab stars becomes strongly flattened at large amplitudes, which was not observed for globular cluster and outer halo field RR Lyr before. We also observed a shift of the two Oosterhoff relations with respect to those of globular clusters. Since the Oosterhoff dichotomy is believed to be caused by RR Lyr stars age differences (about 2 Gyrs for typical globular cluster), and the location on the Bailey diagram can be connected with the formation time, then this could imply different creation scenarios for nearby RR Lyr stars than for globular cluster RR Lyr stars.

The density maps of Oosterhoff groups on the Bailey diagram reveal higher concentrations of stars at certain period and amplitude values, but we do not know whether they are of any significance. However, we could speculate that since the location on the period-amplitude diagram is an indicator of evolutionary state, metallicity or age, prefered certain locations would mean that some states are more probable than the others.

We also put ASAS RRab stars on a period-color diagram. There is no evident dependence of $V-I$ color on period at minimum light, but when using color at 
maximum light two separate groups of stars are visible. These groups are formed by Oosterhoff I and II type variables.

Using methods of Jurcsik and Kovács (1996) and Sandage (2004) we calculated photometric metallicity values for 1008 best quality light curves. We noticed high discrepancies between $[\mathrm{Fe} / \mathrm{H}]$ values from different methods, being also a reflection of the Oosterhoff dichotomy. This suggests that different metallicity relations should be constructed for two Oosterhoff groups, which seems reasonable if we assume that RRab stars of Oosterhoff types are of different age. This observation is supported by comparison of photometric and spectroscopic $[\mathrm{Fe} / \mathrm{H}]$ values.

Comparison of both methods with spectroscopic metallicities from Layden (1994) favors the method of Jurcsik and Kovács (1996) as significantly better for photometric metallicity estimation. The method of Sandage (2004) produces an unphysically bimodal distribution of metallicities which is not seen among RRab stars with spectroscopically obtained $[\mathrm{Fe} / \mathrm{H}]$ values, meaning one can expect high errors in metallicities calculated with this method. Thus the Sandage (2004) method although attractive because of simplicity of its application, should be used with great caution.

The metallicity distributions of ASAS RRab stars show that Oosterhoff II type stars have lower metallicity values, below -1 dex, while Oosterhoff I type stars can be found in the whole metallicity range. The more metal rich, younger stars (Oo I) are observed on average closer to the Galactic plane than older, more metal poor stars (Oo II) as expected, but both Oosterhoff groups are present at all values of $|z|$ implying that the disk is a mixture of young and old RR Lyr stars. However, the increase in metallicity with decreasing $|z|$ is present among both Oosterhoff groups as well as in a short period Oo I subsample, and becomes well defined at $|z| \approx 2.5 \mathrm{kpc}$. We did not observe any significant metallicity trend with distance from the Galactic center, which is in agreement with the results of Kinemuchi et al. (2006).

Acknowledgements. We are thankful to the anonymous referee for useful suggestions. This research has made use of NASA's Astrophysics Data System. The authors were supported by the Polish MNiSW grants N203 007 31/1328 and N N203 304235.

\section{REFERENCES}

Akerlof, C. et al. 2000, Astron. J., 119, 1901.

Bailey, S.I., and Pickering, E.C. 1913, Annals of Harvard College Observatory, 78, 1.

Bono, G., Caputo, F., and Di Criscienzo, M. 2007, Astron. Astrophys., 476, 779.

Cacciari, C., Corwin, T.M., and Carney, B.W. 2005, Astron. J., 129, 267.

Catelan, M. 2009, Astrophys. and Sp. Sci., 320, 261.

Clement, C.M., and Rowe, J.F. 2000, Astron. Astrophys. Suppl. Ser., 196, 4103.

Clement, C.M., and Shelton, I. 1999, Astron. J., 118, 453.

Jurcsik, J., and Kovács, G. 1996, Astron. Astrophys., 312, 111. 
Vol. 59

Kanbur, S.M., and Fernando, I. 2005, MNRAS, 359, 15.

Kinemuchi, K., Smith, H.A., Woźniak, P.R., and McKay, T.A. 2006, Astron. J., 132, 1202.

Kovács, G. 2005, Astron. Astrophys., 438, 227.

Kunder, A., and Chaboyer, B. 2008, Astron. J., 136, 2441.

Layden, A.C. 1994, Astron. J., 108, 1016.

Lee, Y.-W., and Carney, B.W. 1999, Astron. J., 118, 1373.

Miceli, A., et al. 2008, Astrophys. J., 678, 865.

Mizerski, T. 2003, Acta Astron., 53, 307.

Oosterhoff, P.Th. 1939, Observatory, 62, 104.

Pojmański, G. 2002, Acta Astron., 52, 397.

Pojmański, G. 2003, Acta Astron., 53, 341.

Pojmański, G., and Maciejewski, G. 2004, Acta Astron., 54, 153.

Pojmański, G., and Maciejewski, G. 2005, Acta Astron., 55, 97.

Pojmański, G., Pilecki, B., and Szczygieł, D. 2005, Acta Astron., 55, 275.

Sandage, A. 1982, Astrophys. J., 252, 553.

Sandage, A. 2004, Astron. J., 128, 858.

Smolec, R. 2005, Acta Astron., 55, 59.

Soszyński, I., Udalski, A., Szymański, M.K., Kubiak, M., Pietrzyński, G., Wyrzykowski, Ł., Szewczyk, O., Ulaczyk, K., and Poleski, R. 2009, Acta Astron., 59, 1.

Suntzeff, N.B., Kinman, T.D., and Kraft, R.P. 1991, Astrophys. J., 367, 528.

Szczygieł, D.M., and Fabrycky, D.C. 2007, MNRAS, 377, 1263.

van den Bergh, S. 1993, Astron. J., 105, 971.

Vivas, A.K., and Zinn, R. 2003, Memorie della Societa Astronomica Italiana, 74, 928.

Wils, P., Lloyd, C., and Bernhard, K. 2006, MNRAS, 368, 1757.

Woźniak, P.R., et al. 2004, Astron. J., 127, 2436.

Xu, Y., Reid, M.J., Zheng, X.W., and Menten, K.M. 2006, Science, 311, 54.

Zinn, R., and West, M.J. 1984, Astrophys. J. Suppl. Ser., 55, 45. 


\section{Appendix}

\section{Comparison of ASAS and NSVS Catalogs}

\section{The NSVS Project}

The NSVS project was based on data obtained with the Robotic Optical Transient Search Experiment (ROTSE-I) during one year observations between April 1999 and March 2000. ROTSE-I telescopes were located in Los Alamos, New Mexico and scanned the sky north of declination $-38^{\circ}$. The catalog contains stars brighter than unfiltered $15.5 \mathrm{mag}$ (about $15 \mathrm{mag} V$-band equivalent), with about 100-400 good measurements per star. For details on the ROTSE-I experiment refer to Akerlof et al. (2000) and on the NSVS catalog to Woźniak et al. (2004). The NSVS catalog does not provide variability classification itself, but there have been many studies doing so. In particular, RR Lyr type variables have been identified by Kinemuchi et al. (2006) and Wils et al. (2006).

Thanks to the similarity of ASAS and ROTSE telescopes, the ACVS and NSVS catalogs have almost the same sky resolution, detection efficiency, magnitude range and incidence of variable stars, that should allow us to join both databases to create a complete and uniform galactic field RR Lyr stars catalog.

\section{RR Lyr Stars Sample from NSVS}

As already mentioned, there had been at least two independent studies of RR Lyr variables from NSVS, one by Wils et al. (2006), and the other one by Kinemuchi et al. (2006), hereafter K06. The primary difference between them is the selection criteria used to extract the RR Lyr stars sample from NSVS data. Wils et al. (2006) used well known RR Lyr stars from GCVS that are present in NSVS database, determined statistical properties of their light curves and used them to identify all RR Lyr stars in NSVS. The search resulted in 785 RR Lyr stars, 714 of them being RRab Bailey type stars. K06 made use of a cross-correlation of NSVS variable candidates with 2MASS database and used 2MASS colors as one of the selection parameters. This resulted in 1563 RR Lyr stars, among them 1188 RRab stars. In this work we are using the results of K06 for two reasons: because their selection procedure was more similar to the one of ASAS (also using 2MASS color information), and more important, they determined photometric metallicities for $589 \mathrm{RRab}$ variables, being an important RRab stars property investigated in this study.

\section{Combining ASAS and NSVS Samples}

While it is true that both ASAS and NSVS RR Lyr stars data have been obtained with similar equipment, there are several differences that could affect the conformity of both catalogs. First, ROTSE-I observed without filters, thus gathering light in a wide photometric region from $B$ to $I$, and the peak sensitivity being similar to the $R$-band. Given that RR Lyr are blue stars, this could result in not detecting the bluest of them. Second, different algorithms were used to identify and classify RR Lyr stars in the database. 
In order to verify the conformity of the ASAS and NSVS RRab stars samples we investigated variables in an overlapping region, that is for declinations between $-38^{\circ}$ and $+28^{\circ}$. The are 967 ASAS objects and 806 NSVS objects in this area $(806 / 967=83 \%)$, of which only 446 are common $(446 / 967=46 \%$, $446 / 806=55 \%$ ). The first conclusion is that ASAS probably has a better detection efficiency, since it detected more RRab stars than NSVS, while in fact it should detect less - ASAS magnitude range is shifted towards brighter objects with respect to NSVS and the number of stars rises rapidly with larger magnitudes. The reason that NSVS missed a number of objects could be the fact that ROTSE-I observations are made without filters (and only later transformed to their $V$ equivalents) and are less sensitive in the blue regime, occupied by RR Lyr stars. This could be a reason for missing the bluest RR Lyr stars by NSVS.

Another explanation for these high discrepancies would be that RR Lyr stars search and classification algorithms of ASAS and K06 differ significantly, as only about half of objects in each group has their counterparts in the other group (45\% in case of ASAS and 55\% in case of NSVS). As a further check, we want to see how these numbers look for a more conservative sample in the narrower magnitude range.

\section{Comparison of ASAS and NSVS Magnitudes}
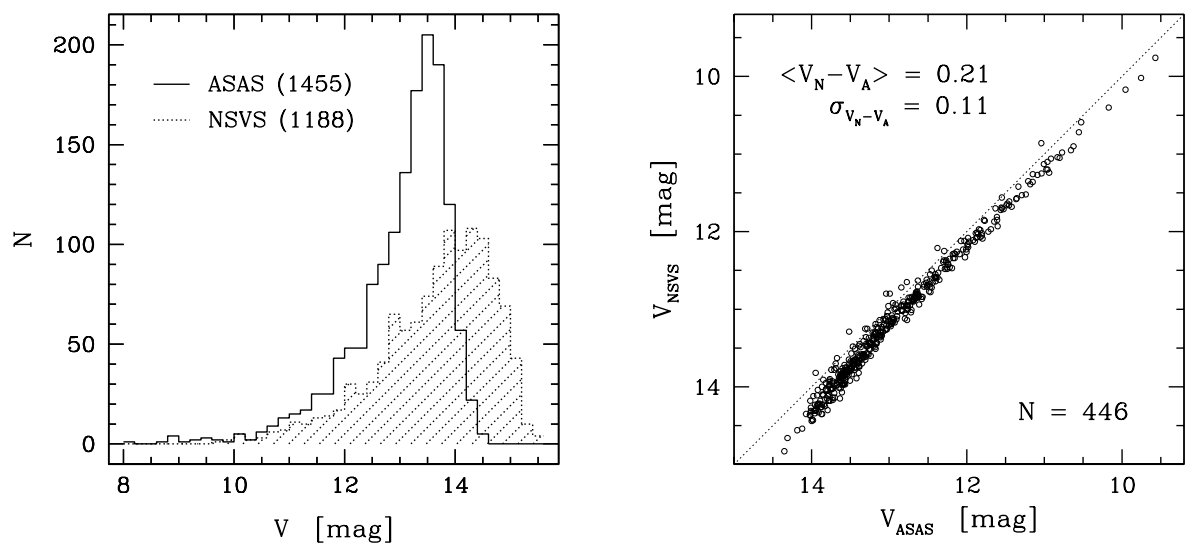

Fig. 18. Left panel shows a histogram of mean magnitudes of all ASAS (plain) and NSVS (shaded) RRab variables. In the right panel there is a comparison of $V$-band magnitudes of ASAS and NSVS counterparts from the overlapping region between declinations $-38^{\circ}$ and $+28^{\circ}$. NSVS magnitudes are on average 0.2 mag larger than their ASAS equivalents.

In order to see RRab stars magnitude distributions, we construct a histogram of mean magnitudes of all RRab stars (the whole sphere) for both samples in the left panel of Fig. 18. It is clear that for a given magnitude bin there are less RRab stars in the NSVS database, and at the same time NSVS observes fainter stars than 
ASAS. This supports the argument that NSVS, while having a larger magnitude limit probably misses the bluest RR Lyr stars due to lower sensitivity in this photometric region.

The $V$-band magnitudes of ASAS and NSVS counterparts from the overlapping region between declinations $-38^{\circ}$ and $+28^{\circ}$ are plotted in the right panel of Fig. 18. The average magnitude difference in star to star comparison is $\left\langle V_{\mathrm{NSVS}}-\right.$ $\left.V_{\mathrm{ASAS}}\right\rangle=0.21 \mathrm{mag}$ and the dispersion is $\sigma_{\left\langle V_{\mathrm{NSVS}}-V_{\mathrm{ASAS}}\right\rangle}=0.11 \mathrm{mag}$. In other words, NSVS magnitudes are on average 0.2 mag higher that their ASAS equivalents. Given this, we shift magnitudes of NSVS sample by 0.21 when combining with the ASAS sample.

Now, we narrow our sample to the magnitude range 10-12 mag. There are 72 NSVS objects and 114 ASAS $(72 / 114=63 \%)$, with 63 common objects (which constitutes $87.5 \%$ for NSVS and $55 \%$ for ASAS). That means, most stars observed by ROTSE-I telescopes are visible for ASAS, while about half of ASAS variables are in the NSVS data. This suggests a better completeness of ASAS catalogs, either due to better sensitivity or more efficient detection procedures.

\section{Comparison of ASAS and NSVS Amplitudes}
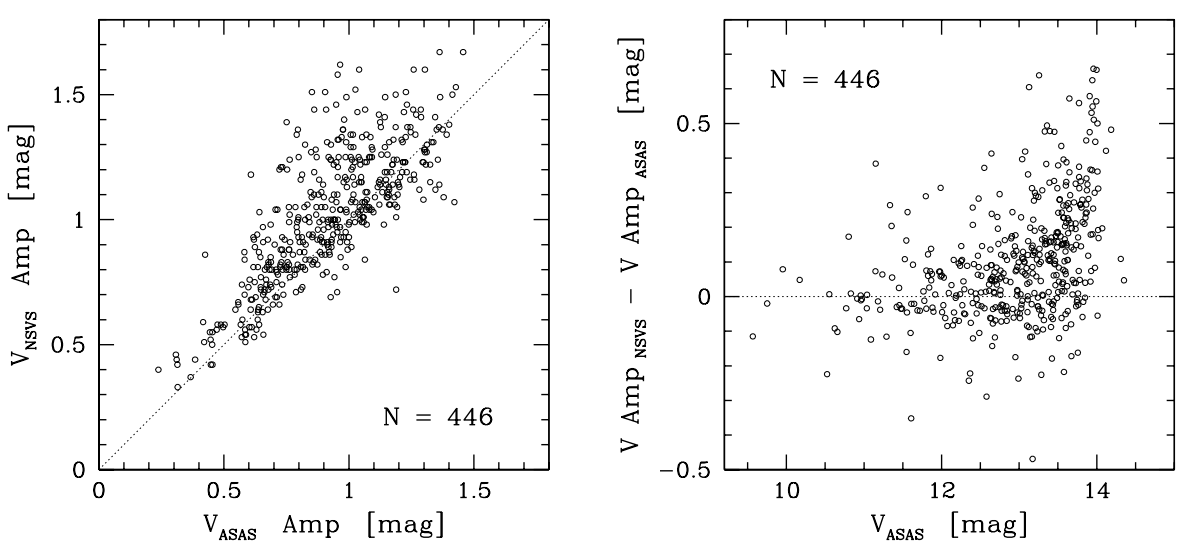

Fig. 19. In the left panel there is a star to star $V$-band amplitude comparison of ASAS and NSVS RRab stars in the overlapping region. NSVS objects have significantly larger amplitudes than their ASAS counterparts and this difference is largest for largest amplitudes. The right panel shows how the amplitude difference depends on the observed magnitude value - the fainter the star, the larger the difference is.

We have also checked the conformity of $V$-band amplitudes for stars from the overlapping region. The left panel of Fig. 19 presents a star to star amplitude comparison. It is clear that the scatter is fairly large, with NSVS amplitudes often much larger than that of ASAS, and the highest scatter is at largest amplitudes. The right panel of the same figure shows how the difference between NSVS and ASAS amplitudes $\left(A_{\mathrm{NSVS}}-A_{\mathrm{ASAS}}\right)$ depends on the magnitude - the fainter the star, the larger 
the difference. It follows, that if we want to combine both samples in the means of amplitudes or any amplitude dependent parameters, we should put the magnitude limit at about 12 mag on both samples (catalogs).

Some amplitude differences at the faint ASAS limit, below $V=13.5$ mag are most probably due to amplitude underestimation for ASAS RRab stars - the faintest points are missed because of the observing limit. But this does not explain all other amplitude differences. We have visually inspected a number of light curves with large amplitude differences and noticed that almost all of them display a high scatter and look like multiperiodic RR Lyr stars. ASAS amplitudes are defined as the magnitude difference between the minimum and maximum of the fitted model, which in these cases will be smaller than the difference between the faintest and the brightest data points. If NSVS amplitudes are calculated in the latter way, this would explain the differences.

\section{Metallicities of NSVS RRab Stars}

K06 calculated metallicities for 589 RRab stars from their original sample of 1188 objects, which had sufficient number of observations and a good phase coverage. They used both methods described in Section 4.1, but the method of JK96 was not applied to a subsample of 175 RRab stars because of large $\varphi_{31}$ errors resulting from a poor Fourier decomposition of the light curves.

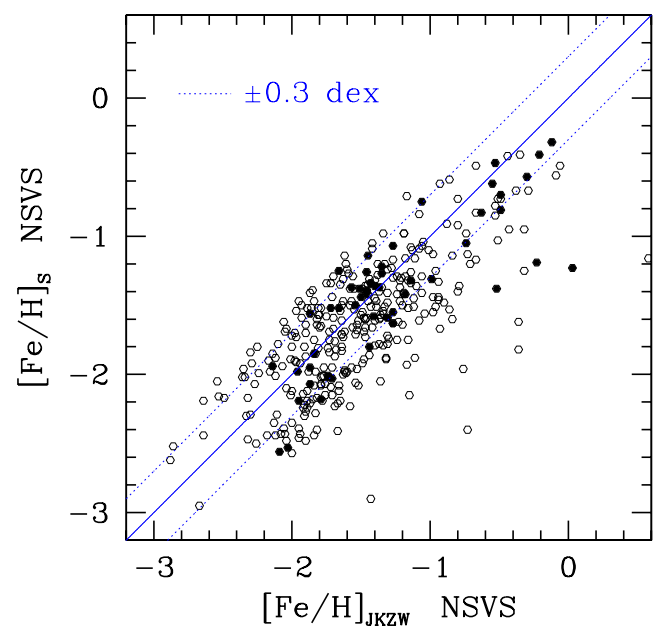

Fig. 20. Comparison of NSVS RRab stars metallicities computed with two methods: JK96 and S04. Filled symbols stand for variables brighter than $12 \mathrm{mag}$.

The comparison of two methods within NSVS sample, similarly to Fig. 10 is presented in Fig. 20. There are 363 RRab stars that have photometric metallicities calculated with both methods. The mean difference is $[\mathrm{Fe} / \mathrm{H}]_{\text {diff }}=[\mathrm{Fe} / \mathrm{H}]_{\mathrm{JKZW}}-$ $[\mathrm{Fe} / \mathrm{H}]_{\mathrm{S}}=0.15$ and the scatter is $\sigma_{[\mathrm{Fe} / \mathrm{H}]_{\text {diff }}}=0.39$. We see that $\mathrm{S} 04$ metallicities are on average lower than JK96 and the shift towards larger $[\mathrm{Fe} / \mathrm{H}]_{\mathrm{JKZW}}$ in the 
region of lower metallicities is observed similarly to Fig. 10. However, there is no clear evidence of the group shifted towards larger $[\mathrm{Fe} / \mathrm{H}]_{\mathrm{S}}$ that is visible in Fig. 10. We suspect this is the result of careful data selection for JK96 method by K06. Nevertheless, we observe a similar discrepancy between methods as was seen in ASAS data (Fig. 10), even though K06 chose only best light curves for JK96 metallicity estimation.

As a final metallicity value they adopted a weighted average of both metallicities for 363 objects and the metallicity of S04 alone for the remaining 226 objects. Among these were 175 stars with large $\varphi_{31}$ errors and a few dozens of stars that had very big differences of metallicities computed from JK96 and S04, even as large as $3 \mathrm{dex}$; for them the $\mathrm{S} 04$ method was assumed to be more reliable.

\section{Comparison of ASAS and NSVS Metallicities}

Now we compare metallicities of ASAS and NSVS RRab stars in the overlapping region. Out of 589 NSVS RRab stars for which the metallicities were obtained 332 stars fall in this area, among them there are 190 objects that have metallicity values from both methods $\left([\mathrm{Fe} / \mathrm{H}]_{\mathrm{S}}\right.$ and $\left.[\mathrm{Fe} / \mathrm{H}]_{\mathrm{JKZW}}\right)$ and 142 with $[\mathrm{Fe} / \mathrm{H}]_{\mathrm{S}}$ only. As shown in Section V NSVS amplitudes are on average bigger than ASAS amplitudes and the fainter the star the bigger the difference. Since the method of S04 depends on amplitude, we should be careful calculating $[\mathrm{Fe} / \mathrm{H}]_{\mathrm{S}}$ when $V>12$ mag. Therefore in the following images we will distinguish a subsample of bright RRab stars with filled symbols.

T a b le 4

Comparison of ASAS and NSVS metallicities

\begin{tabular}{|c|c|c|c|l|c|c|c|}
\hline Method & {$[\mathrm{Fe} / \mathrm{H}]_{\text {diff }}$} & $\sigma_{[\mathrm{Fe} / \mathrm{H}]_{\text {diff }}}$ & $\mathrm{N}_{\text {obs }}$ & & {$[\mathrm{Fe} / \mathrm{H}]_{\text {diff }}$} & $\sigma_{[\mathrm{Fe} / \mathrm{H}]_{\text {diff }}}$ & $\mathrm{N}_{\text {obs }}$ \\
\hline JK96 & -0.02 & 0.50 & 190 & & 0.01 & 0.15 & 124 \\
S04 & 0.09 & 0.42 & 332 & $\left|[\mathrm{Fe} / \mathrm{H}]_{\text {diff }}\right| \leq 0.3$ & 0.06 & 0.13 & 260 \\
JK96 $(V \leq 12)$ & -0.07 & 0.66 & 47 & & 0.03 & 0.13 & 36 \\
S04 $(V \leq 12)$ & -0.01 & 0.38 & 54 & & 0.01 & 0.10 & 48 \\
\hline
\end{tabular}

Star to star comparison of NSVS and ASAS photometric metallicities determined with methods of JK96 and S04. $[\mathrm{Fe} / \mathrm{H}]_{\text {diff }}$ stands for $[\mathrm{Fe} / \mathrm{H}]_{\mathrm{ASAS}}-[\mathrm{Fe} / \mathrm{H}]_{\mathrm{NSVS}}$.

Left panel of Fig. 21 presents a comparison of JK96 metallicities computed in this paper with the ones computed by K06 for NSVS RRab stars. The sample consists of 190 stars. The right panel shows the same comparison for 332 variables with S04 metallicities. The mean difference between ASAS and NSVS values is -0.02 dex for JK96 method and 0.09 dex for S04 and the scatter is 0.50 dex and 0.42 dex respectively. When we take into account objects with $V \leq 12$ mag the average S04 metallicity difference is reduced to -0.01 dex and the dispersion is 0.38 dex. Comparison of the two methods (summarized in Table 4) shows that the method of JK96 produces more consistent results for the whole sample, but it displays quite a big scatter which is mainly the result of a difficulty of $\varphi_{31}$ parameter estimation for distinct light curves from different projects. S04 metallicities are 
on average visibly higher for ASAS than for NSVS as expected, when taking into account the whole sample, but the effect disappears for bright variables.
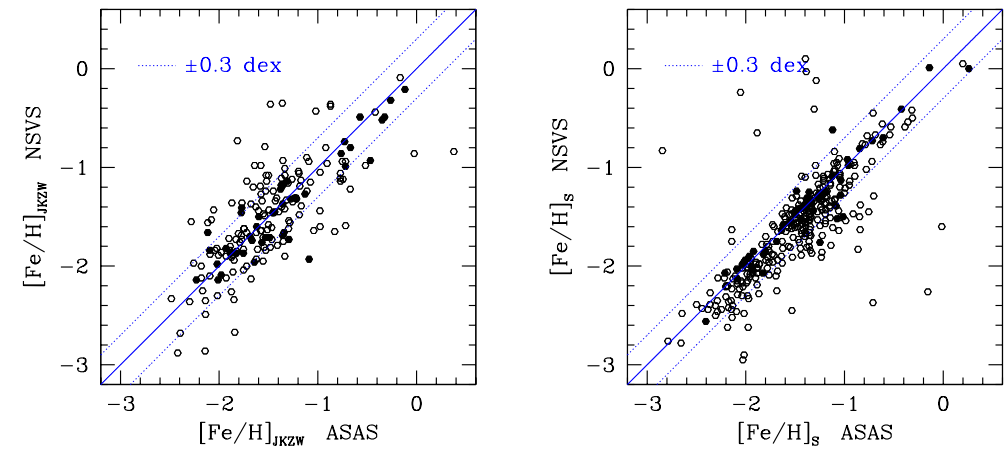

Fig. 21. Comparison of ASAS and NSVS metallicities computed from the method of JK96 (left panel) and S04 (right panel). Filled symbols stand for RRab stars with $V \leq 12$ mag.

Finally, in Fig. 22 we compare NSVS average metallicities with ASAS photometric metallicities. As described in Section 4.5, to determine ASAS photometric metallicities, we used JK96 method, as we believe they are more reliable. The mean difference between ASAS and NSVS is -0.03 dex and the dispersion is 0.66 dex for all 322 objects, while for a subsample of 190 stars the average is 0.04 dex and the dispersion is 0.43 dex. Such large differences in metallicities noticeably affect absolute magnitudes and thus distances: 0.3 dex difference in metallicity produces $50 \mathrm{pc}$ difference in distance for a star of $V=10 \mathrm{mag}$ and $130 \mathrm{pc}$ for a $V=12 \mathrm{mag}$ star.

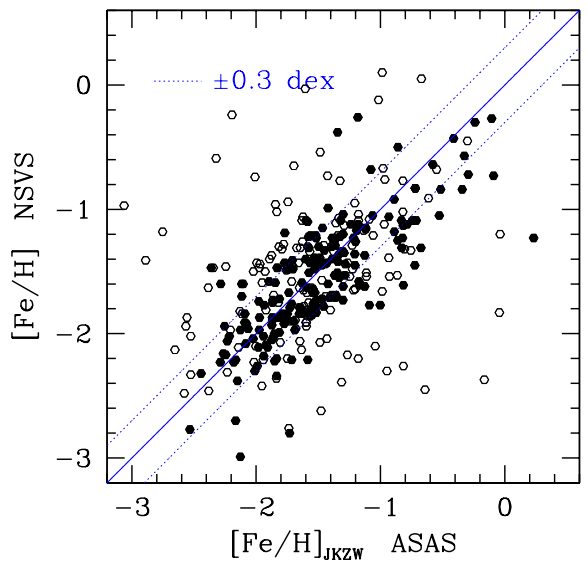

Fig. 22. Comparison of ASAS JK96 metallicities and NSVS best estimate metallicities for 332 RRab stars in the overlapping region. Filled circles stand for 190 RRab stars for which NSVS metallicities from both methods exist. 